\title{
Structural basis of CD4 downregulation by HIV-1 Nef
}

\author{
Yonghwa Kwon', Robyn M. Kaake ${ }^{2,3}$, Ignacia Echeverria ${ }^{4}$, Marissa Suarez ${ }^{5}$, \\ Mohammad Karimian Shamsabadi ${ }^{1}$, Charlotte Stoneham ${ }^{5,6}$, Peter W. Ramirez ${ }^{6}$, Jacob Kress', \\ Rajendra Singh ${ }^{5,6}$, Andrej Sali ${ }^{4,7}$, Nevan Krogan $\circledast^{2,3}$, John Guatelli5,6 and Xiaofei Jia ${ }^{1}{ }^{1 凶}$
}

The HIV-1 Nef protein suppresses multiple immune surveillance mechanisms to promote viral pathogenesis and is an attractive target for the development of novel therapeutics. A key function of Nef is to remove the CD4 receptor from the cell surface by hijacking clathrin- and adaptor protein complex 2 (AP2)-dependent endocytosis. However, exactly how Nef does this has been elusive. Here, we describe the underlying mechanism as revealed by a 3.0- $\AA$ crystal structure of a fusion protein comprising Nef and the cytoplasmic domain of CD4 bound to the tetrameric AP2 complex. An intricate combination of conformational changes occurs in both Nef and AP2 to enable CD4 binding and downregulation. A pocket on Nef previously identified as crucial for recruiting class I MHC is also responsible for recruiting CD4, revealing a potential approach to inhibit two of Nef's activities and sensitize the virus to immune clearance.

T he HIV-1 protein Nef is a critical factor in viral pathogenesis ${ }^{1}$. Expression of Nef in vivo is required for high viral loads and for progression to AIDS2. Individuals infected with HIV-1 encoding defective nef genes do not develop AIDS for decades ${ }^{3,4}$. Nef is a peripheral membrane protein that anchors to the lipid membrane via an $\mathrm{N}$-terminal myristoyl group. Abundantly expressed early during the viral replication cycle, Nef modulates the surface levels of many host proteins and thereby interferes with immune processes responsible for detecting and combating the infection ${ }^{1,5,6}$. Two of the most prominent targets of Nef are MHC-I, which is crucial for antigen presentation and the killing of infected cells by cytotoxic T lymphocytes, and CD4.

CD4 is the entry receptor for HIV-1, but later in the viral replication cycle it is problematic for the virus: it disrupts processing of the viral glycoprotein, Env, inhibiting infectivity ${ }^{7}$; it interferes with the release of new virions ${ }^{8,9}$; and it causes vulnerability to superinfection, causing premature cell death and limiting viral productivity ${ }^{10}$. Furthermore, binding of CD4 to Env exposes otherwise-concealed Env epitopes, rendering infected cells more susceptible to antibody-dependent cellular cytotoxicity and virus particles more susceptible to neutralizing antibodies ${ }^{11-13}$. HIV-1 has evolved strategies to mitigate these problems. Newly synthesized CD4 is targeted in the endoplasmic reticulum by the viral Vpu protein for proteasomal degradation ${ }^{14}$. Surface-expressed CD4, in contrast, is targeted by Nef for endocytosis and lysosomal degradation ${ }^{15-18}$.

Nef's effect on CD4 involves hijacking of clathrin AP2-dependent endocytosis ${ }^{19,20}$. Adaptor protein (AP) complexes mediate the clathrin-dependent trafficking of membrane protein $\mathrm{s}^{21}$. Members of this family (AP1-5) share a common heterotetrameric structure, which adopts either a closed or an open conformation. When activated and open, AP complexes reveal their cargo-bindings sites, which then associate with specific sorting motifs in the cytoplasmic domains of membrane cargos. Two sorting motifs are commonly recognized by APs. The tyrosine-based motifs ( $\operatorname{Yxx} \Phi$, where $\Phi$ is a bulky hydrophobic residue) bind to the $\mu$ subunits of APs, while acidic dileucine motifs ([E/D $] \times x \times L[L / I])$ bind to a pocket formed largely by the $\sigma$ subunits. To mediate downregulation of CD4 from the cell surface, Nef co-opts the clathrin AP2 complex partly by mimicking the acidic dileucine motif ${ }^{22}$. Although how Nef associates with a part of the tetrameric AP2 is understood ${ }^{22}$, to our knowledge, a complete understanding of the interaction, especially how CD4 is sequestered by Nef into a complex with AP2, has remained elusive. Here we describe the mechanism of this key Nef function using a high-resolution crystal structure, cross-linking MS (XL-MS) and structure modeling, along with biochemical and functional validation.

\section{Results}

Nef functions as a 'connector' between clathrin AP2 and CD4. We pursued a high-resolution structure of Nef in complex with tetrameric AP2 and the cytoplasmic domain of $\mathrm{CD} 4\left(\mathrm{CD} 4_{\mathrm{CD}}\right)$. To facilitate the assembly of the protein complex, we fused $\mathrm{CD} 4_{\mathrm{CD}}$ to the $\mathrm{C}$ terminus of Nef via a 36-amino-acid-long, flexible linker (Fig. 1a). Instead of using the full-length CD4 tail (394-433), we included only residues 394-419, containing all of the CD4 determinants reportedly required for Nef-mediated downregulation ${ }^{16,23,24}$. Since Nef residues within the N-terminal amphipathic helix are dispensable for CD4 downregulation ${ }^{25}$, we truncated 25 amino acids from the Nef $\mathrm{N}$ terminus. We engineered the tetrameric AP2 complex by removing the mobile C-terminal domain of the $\mu 2$ subunit (136423), enabling AP2 to adopt an open conformation in which its cargo-binding sites are accessible ${ }^{26}$. Binding between the Nef-CD $4_{\mathrm{CD}}$ fusion protein and the modified AP2 construct (AP2 $\left.{ }^{\triangle \mu 2-C T D}\right)$ was confirmed using a GST pulldown assay (Fig. 1b).

'Department of Chemistry and Biochemistry, University of Massachusetts Dartmouth, Dartmouth, MA, USA. ${ }^{2}$ Department of Cellular and Molecular Pharmacology, University of California, San Francisco, San Francisco, CA, USA. ${ }^{3}$ Gladstone Institutes, San Francisco, CA, USA. ${ }^{4}$ Department of Bioengineering and Therapeutic Sciences, University of California, San Francisco, San Francisco, CA, USA. ${ }^{5}$ The VA San Diego Healthcare System, San Diego, CA, USA. ${ }^{6}$ Department of Medicine, University of California, San Diego, La Jolla, CA, USA. ${ }^{7}$ Department of Pharmaceutical Chemistry and Quantitative Biosciences Institute, University of California, San Francisco, San Francisco, CA, USA. 凶e-mail: xjia@umassd.edu 

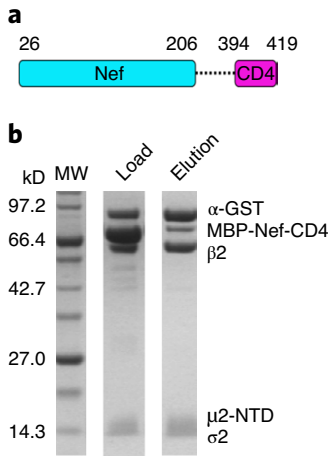
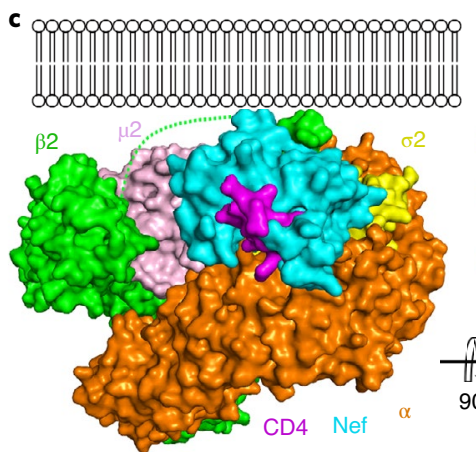
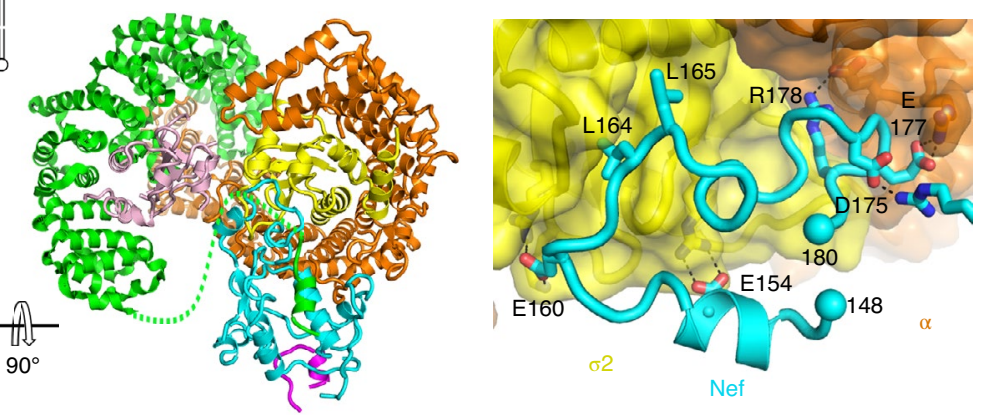

Fig. 1 | In vitro assembly and crystal structure of the Nef, CD4 cytoplasmic domain and clathrin AP2 complex. a, Cartoon illustrating the design of the Nef-CD4 $4_{C D}$ fusion protein. b, In vitro GST pulldown assay confirming the binding between the MBP-Nef-CD4 ${ }_{C D}$ fusion protein and the AP2 ${ }^{\Delta \mu 2-C T D}$ complex. c, Crystal structure of the complete protein complex in two views: along the membrane plane (left) and downward from the membrane (right). $\mathbf{d}$, Nef associates with AP2 mainly through its C-terminal loop (148-180). The rest of Nef is not shown for an unblocked view.

We then solved the crystal structure of the Nef-CD4 $4_{\mathrm{CD}}$ fusion and AP2 ${ }^{\Delta \mu 2-C T D}$ complex to a resolution of $3.0 \AA$ (Fig. $1 \mathrm{c}$ and Table 1 ). All polypeptides are largely resolved except for the flexible linker between Nef and $\mathrm{CD} 4_{\mathrm{CD}}$, which is disordered as expected, and part of the N-terminal region of $\beta 2$. As revealed by the structure, Nef functions as a 'connector' between AP2 and $\mathrm{CD} 4_{\mathrm{CD}}$; $\mathrm{CD} 4_{\mathrm{CD}}$ binds Nef but none of the subunits of AP2. As previously reported, Nef's C-terminal loop interacts with AP2 in part via mimicry of the acidic dileucine motif (Fig. 1d) ${ }^{22}$. The extensive interface here, involving several charge-charge and hydrophobic interactions, is the foundation of the Nef-AP2 association.

CD4 recruitment and role of the Nef N-terminal loop. CD4 is recruited to a pocket on Nef that is opposite the C-terminal loop. The association is mainly hydrophobic and involves three CD4 residues: Ile410, Leu413 and Leu414 (Fig. 2a). The dileucine motif of CD4-Leu413/414-is within a short helix. Leu414 and Ile410 dip into a hydrophobic pocket of Nef, which is formed by Phe121, Trp124, Met79, Thr138 and Pro78 (Fig. 2b). Leu413 of CD4 sits just outside of that pocket and is accommodated by Nef residues Phe121, Leu37, Asn52 and Cys55 (Fig. 2b). Nef residue Asp123 contributes to CD4 binding by hydrogen-bonding with the backbone nitrogen of CD4 Leu413 and supports the helix-turn of CD4 (Fig. 2b). These structural findings explain previously observed roles of CD4 residues-the dileucine motif and Ile410 (refs. ${ }^{16,24,27}$ )as well as Nef residues F121, D123 and Trp124 (refs. ${ }^{28,29}$ ).

CD4 recruitment is secured by the Nef N-terminal loop, which is highly ordered in the structure (Fig. 2c and Extended Data Fig. 1). The part of the loop immediately connected to the rigid core of Nef, residues Phe68 to Pro75, wraps around the core. N-terminally, the loop then takes a sharp 'U-turn', placing a short helix at the interface between the Nef core and the $\alpha$ subunit of AP2. Two residues within this helix, Trp57 and Leu58, fit into a hydrophobic pocket formed mainly by Nef residues: Leu112, Pro122, Phe121 and Tyr115 (Fig. 2d). Lys298 of $\alpha$, with its head group stabilized by Nef Asp111 through charge-charge interactions, also contacts Nef Trp57 via its hydrocarbon chain (Fig. 2d). The Nef acidic cluster (Glu62-65), located in the U-turn region, does not contribute to CD4 binding, consistent with previous functional observations ${ }^{19,30,31}$. The Nef $\mathrm{N}$-terminal loop extends to contact the CD4 tail (Fig. 2c). This part of Nef, residues Cys55 to Val33, forms a wall-like structure to support CD4 binding. Here, contacts are made by several Nef residues with the short helical turn of CD4 and flanking residues. Overall, this conformation of the Nef N-terminal loop explains the crucial roles of Trp57, Leu58, Leu112, Tyr115 and Pro122 in CD4 downregulation observed in previous studies ${ }^{19,20,25,28,32}$. It also explains the

\begin{tabular}{|c|c|}
\hline & Nef-CD4 ${ }_{C D}-A P 2^{\Delta \mu 2-C T D}$ (PDB 6URI) \\
\hline \multicolumn{2}{|l|}{ Data collection ${ }^{\mathrm{a}}$} \\
\hline Space group & $P 4_{1}$ \\
\hline \multicolumn{2}{|l|}{ Cell dimensions } \\
\hline$a, b, c(\AA)$ & $109.2,109.2,178.9$ \\
\hline Wavelength $(\AA)$ & 0.9793 \\
\hline Resolution $(\AA)$ & $47.2(3.11-3.0)^{b}$ \\
\hline$R_{\text {merge }}$ & 0.287 \\
\hline$I / \sigma(I)$ & $11.8(1.4)$ \\
\hline Completeness (\%) & $100(100)$ \\
\hline Redundancy & $16.0(15.9)$ \\
\hline \multicolumn{2}{|l|}{ Refinement } \\
\hline Unique reflections & $41,865(4,189)$ \\
\hline$R_{\text {work }} / R_{\text {free }}$ & $0.241 / 0.277$ \\
\hline \multicolumn{2}{|l|}{ No. atoms } \\
\hline Protein & 12,261 \\
\hline Water & 2 \\
\hline \multicolumn{2}{|l|}{$B$-factors } \\
\hline Protein & 118.30 \\
\hline Water & 132.22 \\
\hline \multicolumn{2}{|l|}{ R.m.s. deviations } \\
\hline Bond lengths $(\AA)$ & 0.005 \\
\hline Bond angles $\left({ }^{\circ}\right)$ & 0.88 \\
\hline \multicolumn{2}{|l|}{ Ramachandran } \\
\hline Favored & $96.42 \%$ \\
\hline Outliers & $0.13 \%$ \\
\hline
\end{tabular}

${ }^{2}$ Data are from one crystal. ${ }^{\circ}$ Values in parentheses are for the highest-resolution shell.

cooperativity in the three-way binding between $\mathrm{CD} 4_{\mathrm{CD}}$, Nef and the $\alpha / \sigma 2$ hemicomplex of AP2 (ref. ${ }^{33}$ ).

We then challenged these structural observations using mutagenesis. First, binding between a fluorescence tag-labeled CD4 cytoplasmic tail (tetramethylrhodamine-CD $4_{\mathrm{CD}}$; TMR-CD $4_{\mathrm{CD}}$ ) and the purified Nef-AP2 complex was characterized by an in vitro fluorescence polarization assay. While unbound TMR-CD $4_{\mathrm{CD}}$ led to 


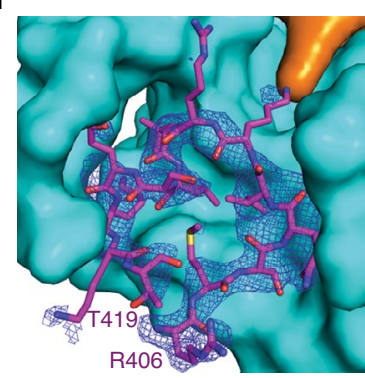

b

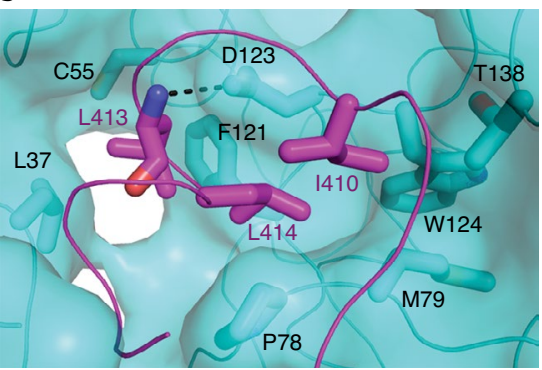

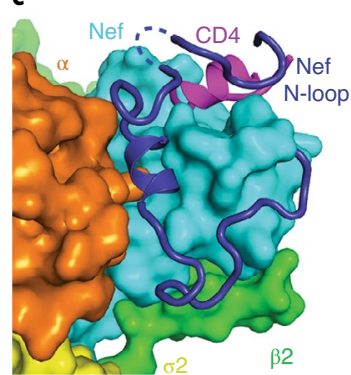

d

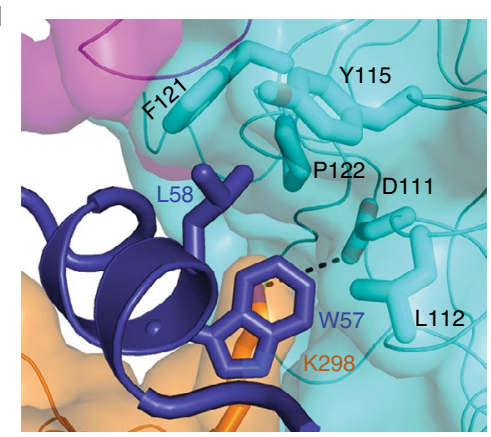

e 7 I $-W T$

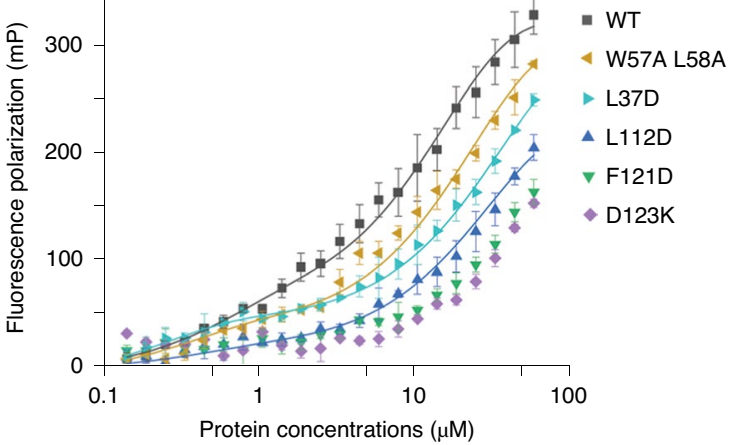

f

$(-)$

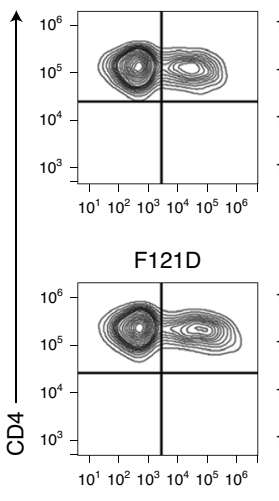

GFP
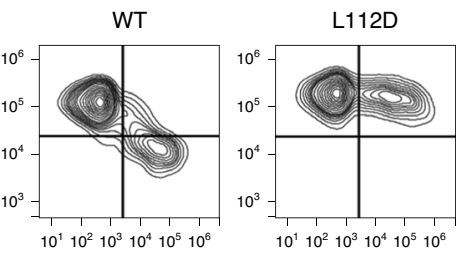

D123R

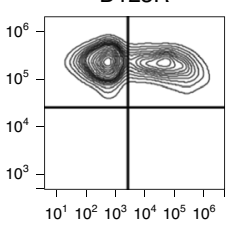

W57A L58A

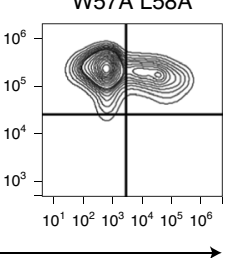

g

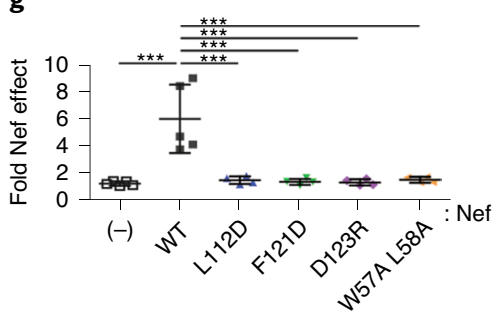

$\mathbf{g}$

h

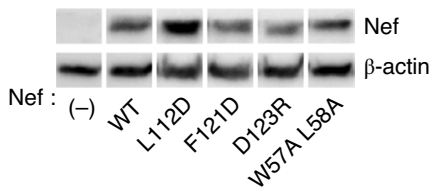

Fig. 2 | Recruitment of CD4 cytoplasmic domain and the role of Nef $\mathbf{N}$-terminal loop. a, A short stretch of the CD4 ${ }_{C D}$ binds to a pocket on Nef. Electron density of the $C D 4_{C D}$ (blue mesh, $2 F_{o}-F_{c}$ map at $1.0 \sigma$ with $B$ factor sharpening of $-50 \AA^{2}$ ) is shown. b, Hydrophobic interactions mediated by CD4 I410, L413 and L414. c, Nef N-terminal loop (dark blue) adopts a unique conformation in the complex. Part of the loop forms a wall of the CD4-binding pocket. Dotted line represents Nef 41-46, which are disordered in the structure. d, Nef residues W57 and L58 mediate the docking of the short helix into the hydrophobic pocket formed at the Nef- $\alpha$ interface. e, Fluorescence polarization assay comparing WT and mutant Nef, when in complex with AP2, for their ability to bind TMR-CD $4_{C D}$. Fluorescence polarization is plotted as a function of Nef concentration (Nef/AP2=1:1.5, molar ratio). Data are shown as mean and s.d. of three technical replicates. f, CD4 downregulation by Nef mutants was measured using cell-surface staining and flow cytometry. CD4-positive HeLa cells were transfected to express Nef and GFP. GFP is a transfection marker; GFP-only control is indicated (-). WT, WT Nef; Nef mutants are indicated. $\mathbf{g}$, Quantification of the fold effect of Nef on the mean fluorescence intensity (MFI) of surface CD4. Data are shown as mean and s.d. for $n=4$ (including L112D, F121D, D123R, W57A L58A mutants) or $n=5$ (including GFP-only control, and WT Nef) independent transfections. Data were compared by one-way analysis of variance with Bonferroni post test; ${ }^{* \star \star} P<0.0001$. h, Expression of Nef proteins in the cells used in the CD4 downregulation assay measured by western blot; the cellular control is $\beta$-actin. Uncropped blot images are shown in Supplementary Fig. 1. Data for graphs in $\mathbf{e}$ and $\mathbf{g}$ are available as source data.

low polarization signal due to its fast tumbling and thus depolarization, binding of TMR-CD4 $4_{\mathrm{CD}}$ to wild-type (WT) Nef and AP2 at higher protein concentrations resulted in drastic increase in molecular size, leading to slower tumbling and thereby high polarization signal (Fig. 2e). In contrast, reduced CD4-binding ability was observed for all Nef mutants tested. These mutations, according to the structure, affect either direct CD4 binding (D123K L37D) or positioning of the Nef N-terminal short helix (W57A L58A L112D) or both (F121D). Second, these same Nef mutations were evaluated in a CD4 surface downregulation assay (Fig. $2 \mathrm{f}-\mathrm{h}$ ).
HeLa cells that stably express CD4 were cotransfected with plasmids expressing GFP (as a transfection marker) and plasmids expressing Nef. Two-color flow cytometry was used to measure cell-surface CD4 as a function of GFP intensity. As expected, WT Nef robustly downregulated CD4. Consistent with the in vitro fluorescence studies, CD4 downregulation was severely impaired for all of the mutants tested (W57A, L58A, L112D, F121D and D123R) (Fig. 2f-h). The L37D mutant was also inactive but poorly expressed (data not shown). Overall, these binding and functional data on the Nef mutants support the structural observations on 


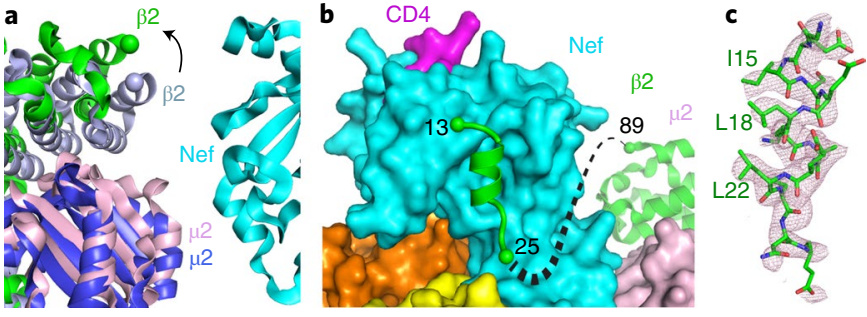

Fig. 3 | Nef binding induces conformational change in the $\beta 2$ subunit of AP2. a, Current structure is overlaid, on the $\alpha-\sigma 2$ half, with the open AP2 structure (PDB 2XA7). $\beta 2$ (green) and $\mu 2$ (pink) subunits in the current structure move outward in comparison with $\beta 2$ (light blue) and $\mu 2$ (blue) subunits of the unbound AP2. b. The first helix of $\beta 2$ binds to the Nef core, while the next three helices become disordered. c, Density for the first helix of $\beta 2$ (pink mesh, $2 F_{0}-F_{c}$ map at $1.0 \sigma$ with $B$ factor sharpening of $-50 \AA^{2}$ ) is shown. Residues important for association with the Nef core are labeled.

CD4 binding and the involvement of the Nef N-terminal loop in the complex.

Nef binding induces a conformational change in the $\beta 2-\mu 2$ half of AP2. Overlaying the $\alpha$ and $\sigma 2$ subunits of the 'open' AP2 structure ${ }^{26}$ on the current structure reveals that the $\beta 2$ subunit and the $\mu 2 \mathrm{~N}$-terminal domain ( $\mu 2-\mathrm{NTD})$ of AP2 in the current structure 'move' out as one rigid body, causing a greater 'opening' of the tetramer (Fig. 3a). In addition, a large portion of the $\beta 2 \mathrm{~N}$ terminus, residues 1-88 encompassing the first four helices, is displaced from the rest of $\beta 2$. If $\beta 2$ had maintained its original fold, then its $\mathrm{N}$ terminus would clash with the Nef core (Extended Data Fig. 2); the observed displacement is likely necessary to accommodate Nef binding. While most of this displaced portion of $\beta 2$ becomes disordered, the first helix preserves its helical structure and binds to the Nef core through hydrophobic interactions (Fig. 3b,c).

The Nef-induced destabilization and structural changes of the $\beta 2-\mu 2$ half of AP2 were further characterized by chemical XL-MS and integrative structure modeling (Extended Data Fig. 3). Here, disuccinimidyl sulfoxide (DSSO), an MS-cleavable, bifunctional amine-reactive small molecule, was used to cross-link proximal Lys residues or $\mathrm{N}$ termini of the Nef-CD $4_{\mathrm{CD}}$ fusion and $\mathrm{AP} 2^{\Delta \mu 2-\mathrm{CTD}}$ complex. Cross-linked proteins separated by SDS-PAGE were trypsin-digested and resulting peptides analyzed by specialized liquid chromatography-MS (LC-MS) ${ }^{3}$ experiments for identification of cross-linked residues (Extended Data Fig. 3a). Application of this pipeline to the complex identified intra- and inter-linked peptides corresponding to 90 unique cross-linked residues (Supplementary Table 1 and Extended Data Fig. 3b). Lys residues from the displaced $\beta 2 \mathrm{~N}$-terminal domain $(1-88)$ are involved in a total of 24 cross-links (Supplementary Table 1, bold entries). Importantly, most of these cross-links are made by Lys residues flanking the first helix (that is, Lys5, 11, 12, 26 and 27), consistent with this helix binding specifically in the complex. In contrast, Lys residues from the other dislocated $\beta 2 \mathrm{~N}$-terminal helices (that is, Lys29, 31, 35, $36,45,66,67$ and 78) are rarely observed, consistent with a lack of fixed residence within the complex. The XL-MS data, which capture structural information from a conformationally heterogeneous population of protein complexes as they exist in solution, the crystal structure and other structural information (Methods) were used for integrative modeling ${ }^{34}$ to produce a model ensemble that describes the complex in full (Extended Data Fig. 3c). Segments that are disordered in the crystal structure are modeled and represented as helices or flexible strings of beads (Extended Data Fig. 3c). The model ensemble agrees satisfactorily with the observed cross-links $(89 \%$ of cross-links are satisfied; Extended Data Fig. 3d). Furthermore, it indicates large variability in the positions and orientations of the helices in the partially unfolded $\beta 2$ segment (Extended Data Fig. $3 \mathrm{e}-\mathrm{g}$ ), consistent with the structural heterogeneity of this region indicated by the crystallographic data.

Nef uses a 'molecular switch' to differentially downregulate MHC-I via AP1 or CD4 via AP2. Binding of the first helix of $\beta 2$ to the Nef core in the current structure closely resembles how the $\mathrm{N}$-terminal amphipathic helix of Nef (deleted from the construct used herein) binds the same location when Nef hijacks AP1 for MHC-I downregulation (Fig. 4a) ${ }^{35}$. That interaction, mediated by Nef Trp13 and Met20, is critical for MHC-I downregulation but is dispensable for CD4 downregulation ${ }^{25,35,36}$. Our current structure indicates that intramolecular association of this Nef helix with the Nef core would force the Nef N-terminal loop to deviate from the conformation associated with CD4 binding (Extended Data Fig. 4). We suspect that this is an intricate plot of the virus: Nef binding forces the $\mathrm{N}$-terminal helices of $\beta 2$ to destabilize and unfold. By providing a binding site for the first $\beta 2$ helix on its core, Nef partially compensates for this destabilization. Moreover, the N-terminal $\beta 2$ helix is now used to compete the Nef amphipathic helix off the core, freeing the $\mathrm{N}$-terminal loop of Nef to adopt the conformation shown in Fig. $2 c$ for CD4 downregulation. A potential caveat to this hypothesis is that the L100A I109A mutation of Nef, which should affect the binding of the $\beta 2$ helix to the Nef-core (Fig. 4a), minimally impairs the downregulation of CD4 (Fig. 4b). However, since this mutation likely affects the binding of the $\beta 2$ helix and the Nef N-terminal amphipathic helix similarly (Fig. 4a), it might have minimal influence on the competition between these two helices for the Nef core in vivo. In contrast, and consistent with the competition hypothesis, the same mutation impairs the downregulation of class I MHC (Fig. 4c), presumably because it disrupts the required binding between the N-terminal amphipathic helix of Nef and the Nef core.

Comparing the current structure with our earlier structure of Nef in complex with the $\mu 1 \mathrm{C}$-terminal domain ( $\mu 1-\mathrm{CTD})$ and the MHC-I cytoplasmic domain reveals versatility and specificity in how the structurally homologous AP1 and AP2 are selectively co-opted to downregulate MHC-I and CD4 (Fig. 4d). For MHC-I downregulation, Nef interacts solely with the $\mu 1$ subunit and exploits the conserved Tyr-based motif-binding site on AP1 (ref. ${ }^{35}$ ). For CD4 downregulation, Nef exploits the acidic dileucine-binding site on the $\alpha$ and $\sigma 2$ subunits of AP2 and contacts all subunits except $\mu 2$. Our models show that by allowing the N-terminal Nef helix and the N-terminal $\beta 2$ helix to bind the same pocket on the core, Nef creates a 'molecular switch' that links its use of different AP complexes with the modulation of different targets: binding to AP2 frees the $\mathrm{N}$ terminus of Nef from the core to recruit CD4, whereas binding to AP1 leaves the core free to bind the $\mathrm{N}$ terminus of $\mathrm{Nef}$ and thereby facilitates the recruitment of MHC-I.

Despite these distinct modes of binding, the cytoplasmic domains of MHC-I and CD4 share much of the same binding 'pocket' on Nef (Fig. 4e). As evident from the structural overlay, the two cytoplasmic domains partially overlap at this location. Notably, Asp123 is the only Nef residue in this pocket that is crucially involved in both binding mechanisms as well as required for the downregulation of both CD4 (refs. ${ }^{28,29}$ ) (Fig. 2b,e-h) and MHC-I (refs. ${ }^{28,29,35-37}$ ). Nonetheless, several residues at this site on Nef are highly conserved (Extended Data Fig. 5).

\section{Discussion}

Our data enable us to postulate the sequence of events leading to the sequestration of CD4 by Nef. Nef first binds to AP2 using its C-terminal loop. This binding, involving the acidic dileucine motif-binding site of AP2, is extensive and robust (Fig. 1d). Unlike typical membrane cargos, which use only short cytoplasmic tails to bind AP2, Nef also contains a bulky core domain, which affixes to 

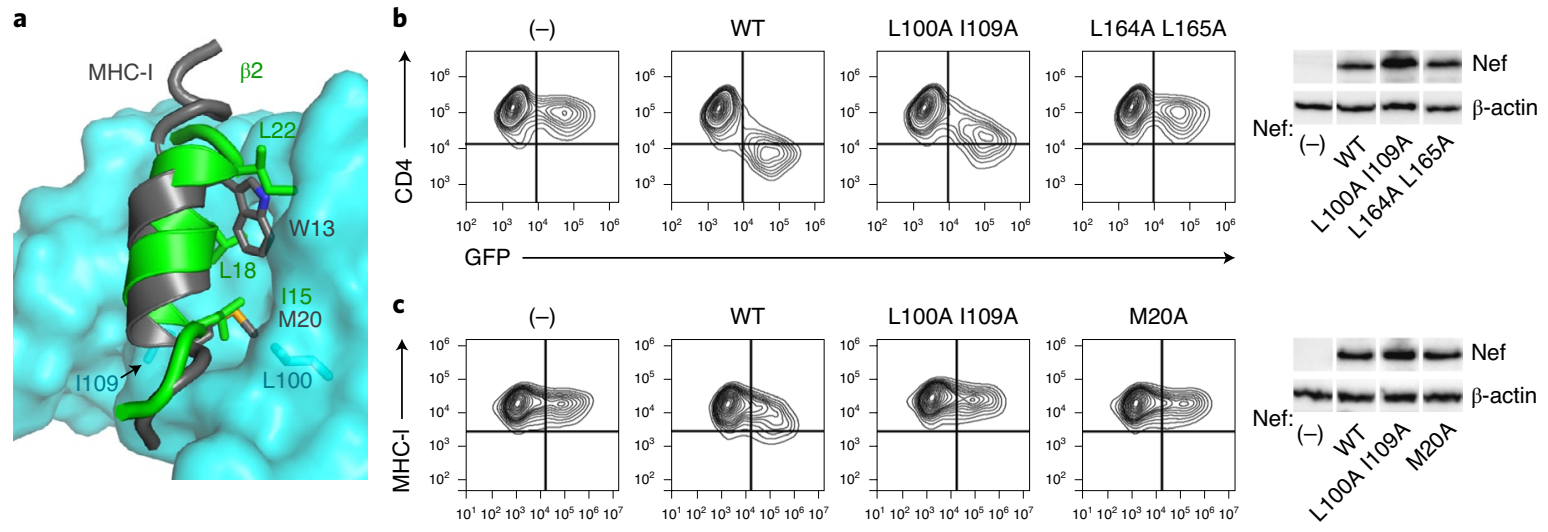

$(-)$
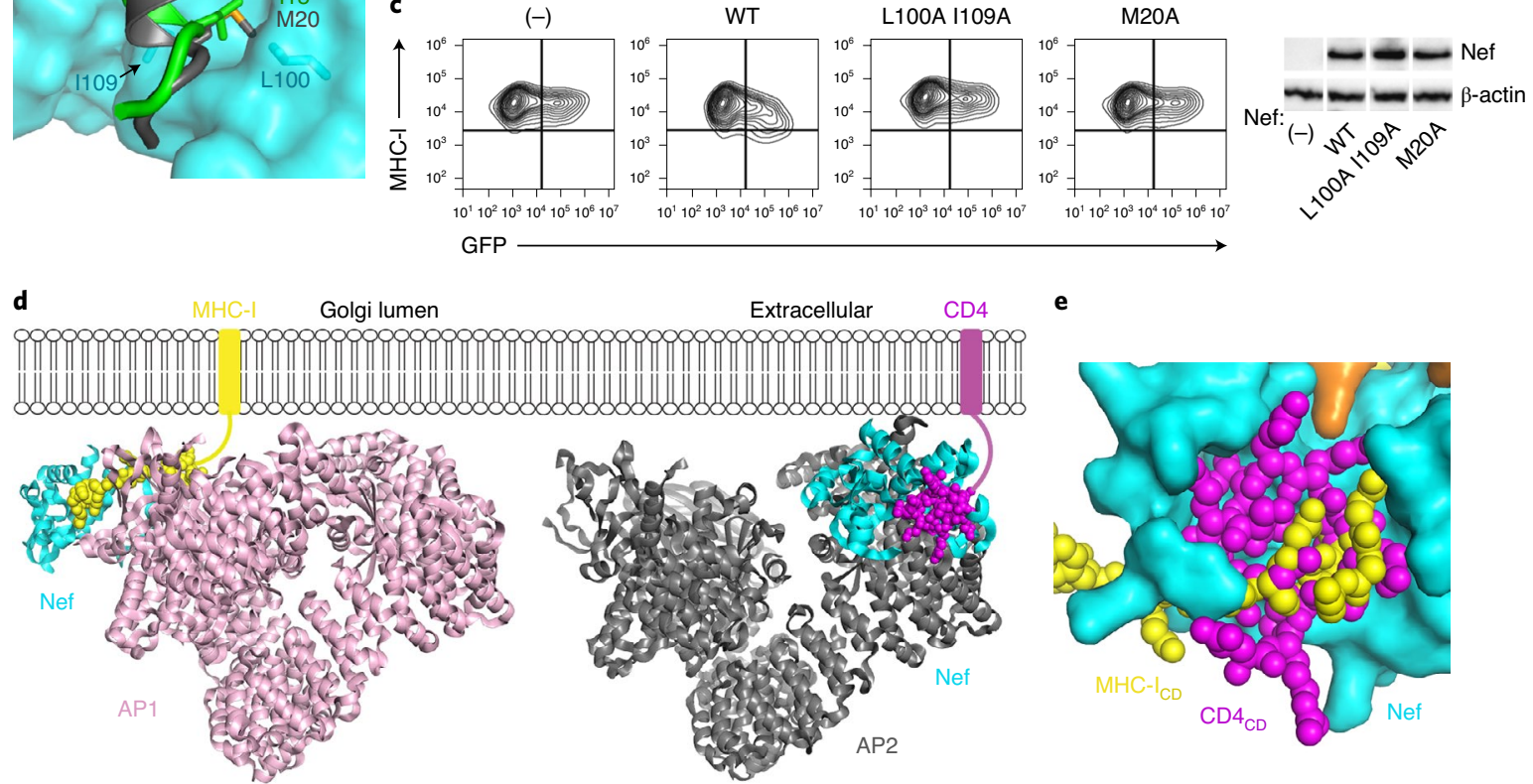

e

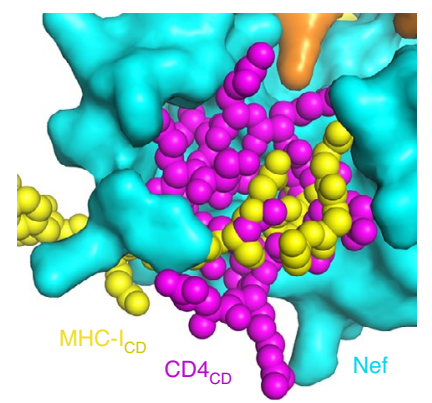

Fig. 4 | The downregulations of MHC-I and CD4 are distinct both mechanistically and structurally, yet their cytoplasmic domains share a common binding site on Nef. a, Nef- $\mu 1-M H C-I_{C D}$ (PDB 4EMZ) and Nef-AP2-CD4 $4_{C D}$ (current structure) are overlaid on Nef (cyan). The Nef N-terminal helix (gray) and the first helix of $\beta 2$ (green, current structure) occupy the same site on the Nef core. Trp13 and Met20 residues in the Nef N-terminal helix important for the intramolecular helix-core association are shown. Important residues on $\beta 2$ (I15, L18 and L22) are also shown. Nef L100 and I109 make direct contact with the bound helices. b,c, CD4 (b) and MHC-I (c) downregulation by Nef mutants was measured using cell-surface staining and flow cytometry. GFP is a transfection marker; GFP-only control is indicated (-). Nef L164A L165A and M20A mutants were used as negative controls for CD4 and MHC-I downregulation, respectively. CD4-positive HeLa cells were used for the CD4 assays, and HEK293 cells, which express HLA-A2, were used for the MHC-I assays. Each experiment was done at least twice (independent transfections). Cellular expression of Nef proteins was compared by western blot; the cellular control is $\beta$-actin. Uncropped blot images are shown in Supplementary Fig. 1. d, Structural comparison of Nef-AP1-MHC-I (PDB 4EMZ; other AP1 subunits modeled based on overlay of the $\mu 1-C T D$ ) and Nef-AP2-CD4 $4_{C D}$ (current structure) shows that Nef hijacks AP1 (left) to downregulate MHC-I and AP2 (right), in turn downregulating CD4 in very different ways. e, Same overlay as in a. Binding of CD4 $4_{C D}$ (magenta) and MHC-I $I_{C D}$ (yellow) involves the same pocket on Nef, yet, with the exception of Nef D123, the specific contact residues are different.

the $\alpha$ and $\sigma 2$ half of AP2. Sterically, the bound Nef core is incompatible with an intact $\beta 2$ subunit (Extended Data Fig. 2). Destabilization of $\beta 2$ then occurs, causing the $\mathrm{N}$-terminal helices to deviate from the bundled state (Fig. 3). The first helix of $\beta 2$ becomes available to bind the Nef core, competing off the N-terminal helix of Nef and thereby freeing the rest of the Nef's N-terminal loop (Fig. 4a). This loop then moves over, placing a short helical anchor at the interface between Nef and the $\alpha$ subunit and extending its arm to complete the CD4-binding site (Fig. 2).

Our results weigh against the notion that dimerization of Nef is required for CD4 downregulation. The dependence of CD4 downregulation on Asp123 has been previously attributed to its role in mediating Nef dimerization ${ }^{28}$. In addition, a quadruple Nef mutant, I109D L112D Y115D F121D (ILYF), which cannot dimerize, is unable to downregulate CD4 (refs. ${ }^{32,38}$ ). However, as revealed here, a single Nef molecule is sufficient to recruit $\mathrm{CD} 4_{\mathrm{CD}}$ into AP2. Moreover, the residues involved in dimerization are directly involved in critical elements of the model here. For example, Asp123 is in direct contact with CD4 (Fig. 2b). Three of the four residues involved in the quadruple mutant, Leu112, Tyr115 and Phe121, interact directly with either CD4 or Nef Trp57/Leu58 (Fig. 2b,d).
Finally, the ILYF-dependent dimerization of Nef, which occurs when Nef is in complex with the Src-family kinase $\mathrm{Hck}^{39}$, is incompatible with the conformation of the Nef N-terminal loop shown here as important for CD4 downregulation (Extended data Fig. 6).

Our results also weigh against the notion that the helix binding site on the Nef core is a promiscuous acceptor for the cytoplasmic domains of target proteins ${ }^{40}$. This hypothesis derived from the observation that this hydrophobic crevice is occupied by the acidic leucine motif from an adjacent Nef molecule in a crystal structure of a simian immunodeficiency virus (SIV) $\mathrm{Nef}^{40}$. Portions of the cytoplasmic domains of CD4 and the CD3 $\zeta$-chain could also be modeled into this site ${ }^{40}$. Our data and model reveal a very different scenario: this hydrophobic crevice on the Nef core binds two distinct helices: a helix from the $\beta 2 \mathrm{~N}$ terminus when in complex with CD4 and AP2, or a helix from the Nef $\mathrm{N}$ terminus when in complex with MHC-I and AP1 (Fig. 4a). As detailed above, this helix binding site on the Nef core is the basis for an elegant molecular switch mechanism that links the use of specific AP complexes with the modulation of different cellular targets.

A key feature of the $\beta 2$ subunit revealed here is that it is structurally labile and, upon Nef binding, can deviate from its original 
fold. Nef-induced destabilization of the $\beta 2$ subunit has also been observed in a recently published structure describing how Nef from an SIV hijacks AP2 to recruit rhesus BST2 for surface downregulation $^{41}$. There, the structural changes at the $\mathrm{N}$ terminus of $\beta 2$ occur in a completely different manner than here. Part of its first helix (14-17) loses its helical fold and instead refolds into a $\beta$-sheet together with a preceding $\beta 2$ segment (6-12) and a segment of Nef's $\mathrm{C}$-terminal loop. This $\beta$-sheet then becomes part of the binding pocket for the recruitment of the rhesus BST2 tail. Whether such a conversion into a $\beta$-sheet occurs when Nef, of either HIV or SIV, targets other cellular surface proteins via AP2-dependent endocytosis is unknown. Similarly, whether the conformational change in $\beta 2$ observed in the present study (Fig. 3) is involved in other downregulation events mediated by Nef remains to be shown. Nonetheless, Nef clearly takes advantage of the destabilization that it causes in $\beta 2$ to induce distinct $\beta 2$ conformations that facilitate recruitment of specific target proteins into clathrin-coated vesicles.

Antiretroviral drugs targeting Nef have the potential to unleash immunologic surveillance mechanisms. Our finding that the structural basis of the downregulation of MHC-I and CD4 involves a common and conserved binding site suggests that both activities could be inhibited by a single agent. Such a Nef inhibitor could facilitate the clearance of infected cells and contribute to HIV cure strategies $^{42}$.

\section{Online content}

Any methods, additional references, Nature Research reporting summaries, source data, extended data, supplementary information, acknowledgements, peer review information; details of author contributions and competing interests; and statements of data and code availability are available at https:/doi.org/10.1038/ s41594-020-0463-z.

Received: 21 January 2020; Accepted: 16 June 2020; Published online: 27 July 2020

\section{References}

1. Kirchhoff, F., Schindler, M., Specht, A., Arhel, N. \& Munch, J. Role of Nef in primate lentiviral immunopathogenesis. Cell. Mol. Life Sci. 65 , 2621-2636 (2008).

2. Kestler, H. W. III et al. Importance of the nef gene for maintenance of high virus loads and for development of AIDS. Cell 65, 651-662 (1991).

3. Kirchhoff, F., Greenough, T. C., Brettler, D. B., Sullivan, J. L. \& Desrosiers, R. C. Brief report: absence of intact Nef sequences in a longterm survivor with nonprogressive HIV-1 infection. N. Engl. J. Med. 332, 228-232 (1995).

4. Deacon, N. J. et al. Genomic structure of an attenuated quasi-species of HIV-1 from a blood-transfusion donor and recipients. Science 270, 988-991 (1995).

5. Pereira, E. A. \& daSilva, L. L. HIV-1 Nef: taking control of protein trafficking. Traffic 17, 976-996 (2016).

6. Pawlak, E. N. \& Dikeakos, J. D. HIV-1 Nef: a master manipulator of the membrane trafficking machinery mediating immune evasion. Biochim. Biophys. Acta 1850, 733-741 (2015).

7. Willey, R. L., Maldarelli, F., Martin, M. A. \& Strebel, K. Human immunodeficiency virus type $1 \mathrm{Vpu}$ protein regulates the formation of intracellular gp160-CD4 complexes. J. Virol. 66, 226-234 (1992).

8. Ross, T. M., Oran, A. E. \& Cullen, B. R. Inhibition of HIV-1 progeny virion release by cell-surface CD4 is relieved by expression of the viral Nef protein. Curr. Biol. 9, 613-621 (1999).

9. Lama, J., Mangasarian, A. \& Trono, D. Cell-surface expression of CD4 reduces HIV-1 infectivity by blocking Env incorporation in a Nef- and Vpu-inhibitable manner. Curr. Biol. 9, 622-631 (1999).

10. Benson, R. E., Sanfridson, A., Ottinger, J. S., Doyle, C. \& Cullen, B. R. Downregulation of cell-surface CD4 expression by simian immunodeficiency virus Nef prevents viral super infection. J. Exp. Med. 177, 1561-1566 (1993).

11. Veillette, M. et al. Interaction with cellular CD4 exposes HIV-1 envelope epitopes targeted by antibody-dependent cell-mediated cytotoxicity. J. Virol. 88, 2633-2644 (2014).

12. Pham, T. N., Lukhele, S., Hajjar, F., Routy, J. P. \& Cohen, E. A. HIV Nef and Vpu protect $\mathrm{HIV}$-infected $\mathrm{CD} 4^{+} \mathrm{T}$ cells from antibody-mediated cell lysis through down-modulation of CD4 and BST2. Retrovirology 11, 15 (2014)
13. Ding, S. et al. CD4 incorporation into HIV-1 viral particles exposes envelope epitopes recognized by CD4-induced antibodies. J. Virol. 93, e01403-19 (2019).

14. Schubert, U. et al. CD4 glycoprotein degradation induced by human immunodeficiency virus type $1 \mathrm{Vpu}$ protein requires the function of proteasomes and the ubiquitin-conjugating pathway. J. Virol. 72, 2280-2288 (1998).

15. daSilva, L. L. P. et al. Human immunodeficiency virus type 1 Nef protein targets CD4 to the multivesicular body pathway. J. Virol. 83, 6578-6590 (2009).

16. Aiken, C., Konner, J., Landau, N. R., Lenburg, M. E. \& Trono, D. Nef induces CD4 endocytosis-requirement for a critical dileucine motif in the membrane-proximal Cd4 cytoplasmic domain. Cell 76, 853-864 (1994).

17. Garcia, J. V. \& Miller, A. D. Serine phosphorylation-independent down-regulation of cell-surface CD4 by nef. Nature 350, 508-511 (1991).

18. Guy, B. et al. HIV F/3' orf encodes a phosphorylated GTP-binding protein resembling an oncogene product. Nature 330, 266-269 (1987).

19. Chaudhuri, R., Lindwasser, O. W., Smith, W. J., Hurley, J. H. \& Bonifacino, J. $\mathrm{S}$. Downregulation of CD4 by human immunodeficiency virus type $1 \mathrm{Nef}$ is dependent on clathrin and involves direct interaction of Nef with the AP2 clathrin adaptor. J. Virol. 81, 3877-3890 (2007).

20. Greenberg, M. E. et al. Co-localization of HIV-1 Nef with the AP-2 adaptor protein complex correlates with Nef-induced CD4 down-regulation. EMBO J. 16, 6964-6976 (1997)

21. Traub, L. M. \& Bonifacino, J. S. Cargo recognition in clathrin-mediated endocytosis. Cold Spring Harb. Perspect. Biol. 5, a016790 (2013).

22. Ren, X., Park, S. Y., Bonifacino, J. S. \& Hurley, J. H. How HIV-1 Nef hijacks the AP-2 clathrin adaptor to downregulate CD4. Elife 3, e01754 (2014).

23. Preusser, A., Briese, L., Baur, A. S. \& Willbold, D. Direct in vitro binding of full-length human immunodeficiency virus type 1 Nef protein to CD4 cytoplasmic domain. J. Virol. 75, 3960-3964 (2001).

24. Salghetti, S., Mariani, R. \& Skowronski, J. Human immunodeficiency virus type $1 \mathrm{Nef}$ and $\mathrm{p} 56^{\mathrm{lck}}$ protein-tyrosine kinase interact with a common element in CD4 cytoplasmic tail. Proc. Natl Acad. Sci. USA 92, 349-353 (1995).

25. Mangasarian, A., Piguet, V., Wang, J. K., Chen, Y. L. \& Trono, D. Nef-induced CD4 and major histocompatibility complex class I (MHC-I) down-regulation are governed by distinct determinants: $\mathrm{N}$-terminal alpha helix and proline repeat of Nef selectively regulate MHC-I trafficking. J. Virol. 73, 1964-1973 (1999)

26. Jackson, L. P. et al. A large-scale conformational change couples membrane recruitment to cargo binding in the AP2 clathrin adaptor complex. Cell 141, 1220-U213 (2010).

27. Hua, J. \& Cullen, B. R. Human immunodeficiency virus types 1 and 2 and simian immunodeficiency virus Nef use distinct but overlapping target sites for downregulation of cell surface CD4. J. Virol. 71, 6742-6748 (1997).

28. Liu, L. X. et al. Mutation of a conserved residue (D123) required for oligomerization of human immunodeficiency virus type 1 Nef protein abolishes interaction with human thioesterase and results in impairment of Nef biological functions. J. Virol. 74, 5310-5319 (2000).

29. Hua, J., Blair, W., Truant, R. \& Cullen, B. R. Identification of regions in HIV-1 Nef required for efficient downregulation of cell surface CD4. Virology 231, 231-238 (1997).

30. Baugh, L. L., Garcia, J. V. \& Foster, J. L. Functional characterization of the human immunodeficiency virus type 1 Nef acidic domain. J. Virol. 82, 9657-9667 (2008)

31. Aiken, C., Krause, L., Chen, Y. L. \& Trono, D. Mutational analysis of HIV-1 Nef: identification of two mutants that are temperature-sensitive for CD4 downregulation. Virology 217, 293-300 (1996).

32. Poe, J. A. \& Smithgall, T. E. HIV-1 Nef dimerization is required for Nef-mediated receptor downregulation and viral replication. J. Mol. Biol. 394, 329-342 (2009)

33. Chaudhuri, R., Mattera, R., Lindwasser, O. W., Robinson, M. S. \& Bonifacino, J. S. A basic patch on a-adaptin is required for binding of human immunodeficiency virus type $1 \mathrm{Nef}$ and cooperative assembly of a CD4-Nef-AP-2 complex. J. Virol. 83, 2518-2530 (2009).

34. Rout, M. P. \& Sali, A. Principles for integrative structural biology studies. Cell 177, 1384-1403 (2019).

35. Jia, X. et al. Structural basis of evasion of cellular adaptive immunity by HIV-1 Nef. Nat. Struct. Mol. Biol. 19, 701-706 (2012).

36. Williams, M., Roeth, J. F., Kasper, M. R., Filzen, T. M. \& Collins, K. L. Human immunodeficiency virus type $1 \mathrm{Nef}$ domains required for disruption of major histocompatibility complex class I trafficking are also necessary for coprecipitation of Nef with HLA-A2. J. Virol. 79, 632-636 (2005).

37. Wonderlich, E. R., Williams, M. \& Collins, K. L. The tyrosine binding pocket in the adaptor protein $1(\mathrm{AP}-1) \mu 1$ subunit is necessary for Nef to recruit AP-1 to the major histocompatibility complex class I cytoplasmic tail. J. Biol. Chem. 283, 3011-3022 (2008).

38. Shu, S. T., Emert-Sedlak, L. A. \& Smithgall, T. E. Cell-based fluorescence complementation reveals a role for HIV-1 Nef protein dimerization in AP-2 adaptor recruitment and CD4 co-receptor down-regulation. J. Biol. Chem. 292, 2670-2678 (2017). 
39. Alvarado, J. J., Tarafdar, S., Yeh, J. I. \& Smithgall, T. E. Interaction with the Src homology (SH3-SH2) region of the Src-family kinase Hck structures the HIV-1 Nef dimer for kinase activation and effector recruitment. J. Biol. Chem. 289, 28539-28553 (2014).

40. Manrique, S. et al. Endocytic sorting motif interactions involved in Nef-mediated downmodulation of CD4 and CD3. Nat. Commun. 8 442 (2017)
41. Buffalo, C. Z. et al. Structural basis for Tetherin Antagonism as a Barrier to Zoonotic Lentiviral Transmission. Cell Host Microbe 26, 359-368.e8 (2019). 42. Deeks, S. G. HIV: shock and kill. Nature 487, 439-440 (2012).

Publisher's note Springer Nature remains neutral with regard to jurisdictional claims in published maps and institutional affiliations.

(c) The Author(s), under exclusive licence to Springer Nature America, Inc. 2020 


\section{Methods}

Materials. The gene of rat $\alpha$ adaptin was a kind gift from J.Bonifacino (National Institutes of Health). Genes of $\beta 2, \mu 2$ and $\sigma 2$ adaptins were amplified from a complementary DNA library of human HEK293T cells. For XL-MS analysis, anhydrous DMSO, DSSO, MS-grade trypsin, HPLC-grade water, formic acid and acetonitrile were all purchased from Thermo Fisher Scientific. The 4-20\% TGX SDS-PAGE gels were purchased from Bio-Rad. MS-safe AcquaStain was purchased from Bulldog Bio.

Cloning, expression and purification of proteins. The Nef-CD $4_{\mathrm{CD}}$ fusion was constructed by fusing CD4 (394-419) to the C terminus of HIV-1 Nef (26-206, NL4.3) via a flexible linker of 36 amino acids (GVDGSDEASELACPTPKEDGL AQQQTQLNLRGSGSG). The encoding gene was cloned into a pMAT9 expression vector. The fusion protein was over-expressed in Escherichia coli NiCo21(DE3) cells, carrying an N-terminal maltose binding protein (MBP) tag. Cells were induced with $0.1 \mathrm{mM}$ IPTG at an optical density at $600 \mathrm{~nm}$ of 0.8 and grown at $16^{\circ} \mathrm{C}$ overnight. Cells were lysed using sonication. Expressed protein was first purified using the MBP affinity column. For GST pulldown experiments, the MBP-tagged protein was further purified by a HiTrap Q anion exchange column, followed by a final Superdex 200 size exclusion column. For crystallization, the MBP fusion tag was cleaved off by the SARS-CoV $\mathrm{M}^{\text {pro }}$ protease ${ }^{43,44}$ after the affinity purification. The tagless protein was similarly purified using the HiTrap Q anion exchange column and the Superdex 200 size exclusion column. WT and mutant Nef proteins used for the fluorescence polarization assays were produced similarly.

For AP2 $2^{\Delta \mu 2-C T D}$, genes for each of the four subunits were cloned into two duet vectors: a pETDuet vector with human $\beta 2(1-591)$ and human $\mu 2(1-135)$ at each of the multiple cloning sites, respectively; and a pCDFDuet vector with rat $\alpha(1-621)$ carrying a C-terminal GST tag and human $\sigma 2(1-142)$. The heterotetrameric $\mathrm{AP} 2^{\Delta 42-\mathrm{CTD}}$ core was expressed overnight at $22^{\circ} \mathrm{C}$ in the NiCo21(DE3) cells in Terrific broth after induction with IPTG. For binding assays, the GST-tagged AP2 ${ }^{\triangle \mu 2-C T D}$ complex was purified by a sequence of Ni-nitrilotriacetic acid (Ni-NTA) gravity column, GST affinity column and Superdex 200 size exclusion column. For crystallization, the GST tag was cleaved off by the Tev protease after the GST affinity purification, followed by a final purification using the Superdex 200 size exclusion column.

In vitro GST pulldown assay. Purified proteins AP2 $2^{\Delta \mu 2-C T D}-G S T(0.2 \mathrm{mg})$ and MBP-Nef-CD4 $4_{\mathrm{CD}}(0.4 \mathrm{mg})$ were mixed in a final volume of $100 \mu \mathrm{l}$ and incubated at $4{ }^{\circ} \mathrm{C}$ for $30 \mathrm{~min}$. The protein solution was then loaded onto a small gravity flow column containing $0.2 \mathrm{ml}$ of GST resin. Flow-through was collected and the resin was extensively washed with $5 \times 0.9 \mathrm{ml}^{2}$ of GST binding buffer $(50 \mathrm{mM}$ Tris, $\mathrm{pH} 8,100 \mathrm{mM} \mathrm{NaCl}, 0.1 \mathrm{mM}$ TCEP). The bound proteins were then eluted with $5 \times 0.1 \mathrm{ml}^{2}$ of GST elution buffer containing $10 \mathrm{mM}$ reduced glutathione. The eluted proteins were analyzed by SDS-PAGE.

Fluorescence polarization assay. Purified MBP-Nef and AP2 ${ }^{\Delta \mu 2-\mathrm{CTD}}$ proteins were buffer exchanged, respectively, into the assay buffer $(50 \mathrm{mM}$ sodium phosphate, $\mathrm{pH}$ $6.5,5 \mathrm{mM} \mathrm{KCl}, 2 \mathrm{mM}$ dithiothreitol). A stock protein solution was then prepared containing $80 \mu \mathrm{M}$ MBP-Nef and $120 \mu \mathrm{M}$ AP2 $2^{\Delta \mu 2-C T D}$, which was subsequently used to create different dilutions. Assays were carried out in Corning 384-well black microplates (3820). In each well, $200 \mathrm{nM}$ TMR-labeled CD4 peptide (CD4 408-417, synthesized by GenScript) was mixed with MBP-Nef and AP2 $2^{\triangle \mu 2-C T D}$ at varied concentrations in a total volume of $50 \mu \mathrm{l}$. Incubation was carried out for $1 \mathrm{~h}$ at room temperature with minimal exposure to light. Fluorescence polarization was then measured using the EnVision plate reader (Perkin Elmer) with excitation at $535 \mathrm{~nm}$ and emission at $595 \mathrm{~nm}$. Fluorescence polarization measurement was repeated three times and plotted as a function of protein concentration in a logarithmic scale using Origin.

Crystallization and crystallographic data collection. Crystallization was carried out using the microbatch under-oil method. The purified AP2 ${ }^{\Delta \mu 2-C T D}$ core and the Nef-CD $4_{\mathrm{CD}}$ fusion chimera were mixed at 1:5 molar ratio to a final concentration of $2.5 \mathrm{mg} \mathrm{ml}^{-1}$ (25 mM Tris, pH 8.0, $100 \mathrm{mM} \mathrm{NaCl}, 0.1 \mathrm{mM}$ TCEP, $0.1 \mathrm{mM}$ PMSF). Equal volumes of the protein solution and the precipitant solution $(100 \mathrm{mM}$ HEPES, pH 6.9, $200 \mathrm{mM} \mathrm{KCl,} \mathrm{15 \%} \mathrm{PEG4000,} \mathrm{6 \%} \mathrm{1,6-hexanediol)} \mathrm{were} \mathrm{mixed.} \mathrm{The}$ drop was sealed using a mixture of paraffin and silicon oil at a 2:1 ratio. Crystals appeared within $24 \mathrm{~h}$ at room temperature and grew to full size in about a week.

Crystals were cryo-protected using the precipitant solution containing $20 \%$ glycerol and then frozen in liquid nitrogen. Datasets were collected at the Northeastern Collaborative Access Team (NE-CAT, 24-ID) at the Advanced Photon Source, Argonne National Laboratory, and the Frontier Microfocusing Macromolecular Crystallography (FMX, 17-ID-2) and Highly Automated Macromolecular Crystallography (AMX, 17-ID-1) at the National Synchrotron Light Source II, Brookhaven National Laboratory. Diffraction data were processed using HKL2000 (ref. ${ }^{45}$ ). The crystals were in the P41 space group and diffracted to a highest resolution of $3.0 \AA$. The statistics are summarized in Table 1.

Structure determination and refinement. The structural solution was obtained by molecular replacement using PHASER ${ }^{46}$ in Phenix ${ }^{47}$. Only one molecule exists in the asymmetric unit. The Protein Data Bank (PDB) identity of the open AP2 core (2XA7) was divided into two search models: the $\alpha$ and $\sigma 2$ hemicomplex and the $\beta 2$ and $\mu 2$-NTD hemicomplex. Together with the Nef structure (4EMZ), the three models were used sequentially to successfully obtain the solution. Iterative rounds of model building in COOT $^{48}$ and refinement in Phenix ${ }^{49}$ were carried out. The final model has an $R_{\text {work }} / R_{\text {free }}$ of $0.241 / 0.277$. A Ramachandran plot showed that $96.4 \%$ of the residues are in the favored region, together with $3.5 \%$ in the allowed region and $0.1 \%$ as outliers. The refinement statistics are summarized in Table 1.

CD4 downregulation assays. HeLa cells expressing CD4 (TZM-bl, obtained from Dr. John Kappes via the National Institutes of Health AIDS Reagent Program) were transfected using Lipofectamine2000 (Thermo Fisher Scientific) with pCG-GFP (a gift from Dr. Jacek Skowronski) and pCI-NL, a pCI-neo-based plasmid (Promega) expressing $\mathrm{Nef}_{\mathrm{NL} 4-3}$ or the indicated Nef-mutants. In each transfection, $1.6 \mu \mathrm{g}$ of total plasmid DNA was used: $0.4 \mu \mathrm{g}$ of pCG-GFP and $1.2 \mu \mathrm{g}$ of pCI-NL or derivative mutants. One of the replicate experiments was done using Fugene 6 (Promega) as the transfection reagent, $0.3 \mu \mathrm{g}$ of pCG-GFP and $0.9 \mu \mathrm{g}$ of pCI-NL or derivative mutants. After $1 \mathrm{~d}$, half of the cells were stained for surface CD4 (anti-human CD4, BioLegend, conjugated directly to APC), fixed in formaldehyde, then analyzed by two-color flow cytometry using an Accuri 6 flow cytometer (BD). 'Live cell' gates were set using untransfected cells; gates for GFP were set using cells transfected only with pCI-neo; and gates for CD4 were set using cells stained with an APC-conjugated antibody isotype control. Two-color contour plots were prepared using FlowJo Software (BD), v.10.6.1. The other half of the cells were lysed in Laemmli buffer, and the proteins were resolved on $10 \%$ denaturing SDS-PAGE gels before transfer to PVDF membranes. Nef was detected using a polyclonal antiserum raised to NL4-3 Nef in sheep (a gift from Dr. Celsa Spina, University of California, San Diego). $\beta$-actin was detected using a murine monoclonal antibody (Sigma-Aldrich). Species-specific secondary antibodies conjugated to HRP were visualized using Western Clarity detection reagent (Bio-Rad). Chemiluminescence was recorded using a ChemiDoc Imager System (Bio-Rad). The cells were tested for mycoplasma using a PCR assay and were negative.

Class I MHC downregulation assays. HEK293 cells, which naturally express HLA-A2, were transfected using Lipofectamine2000 with pCG-GFP $(0.4 \mu \mathrm{g})$ and pCI-NL, the indicated Nef mutants or the empty vector pCI-neo (1.2 $\mu \mathrm{g})$. After $1 \mathrm{~d}$, half of the cells were stained for surface HLA-A2 (BioLegend, anti-HLA-A2 conjugated directly to APC), fixed in formaldehyde, then analyzed by two-color flow cytometry using an Accuri 6 flow cytometer. 'Live cell' gates were set using untransfected cells; gates for GFP were set using cells transfected only with pCI-neo; and gates for HLA-A2 were set using cells stained with an APC-conjugated antibody isotype control. Two-color contour plots were prepared using FlowJo Software (BD), v.10.6.1. The other half of the cells were processed for western blot as described in the CD4 downregulation assays. The cells were tested for mycoplasma using a PCR assay and were negative.

DSSO-based XL-MS analysis. Individual preparations of Nef-CD $4_{\mathrm{CD}}-\mathrm{AP} 2^{\Delta \mu 2-\mathrm{CTD}}$ complex $\left(2.8 \mathrm{mg} \mathrm{ml}^{-1}\right.$ and $0.7 \mathrm{mg} \mathrm{ml}^{-1}$ at $1: 5 \mathrm{Nef}-\mathrm{CD} 4_{\mathrm{CD}}$ molar excess) were cross-linked using increasing molar ratios of DSSO (Thermo Fisher Scientific), for 5,10 or $30 \mathrm{~min}$ at 4 or $37^{\circ} \mathrm{C}$. Cross-linked proteins were separated on $4-20 \%$ TGX gradient SDS-PAGE gels (Bio-Rad) and stained with MS-safe AcquaStain (Bulldog Bio); then, cross-linked product bands were excised and submitted for in-gel reduction, alkylation and trypsin digestion. Extracted peptides were separated online by Thermo Easy nLC 1000 by reverse-phase HPLC $(75 \mu \mathrm{m} \times 30 \mathrm{~cm}$ fused silica packed with 1.9- $\mu \mathrm{m}$ Reprosil-Pur C18 AQ resin (Dr. Maisch-GmbH) column), running a linear gradient of $5-30 \% \mathrm{~B}$ in $50 \mathrm{~min}, 35-95 \% \mathrm{~B}$ in $5 \mathrm{~min}$ and $95 \% \mathrm{~B}$ for $4 \mathrm{~min}$ at a flow rate of $300 \mathrm{nl} \mathrm{min}^{-1}$ (buffer A: $100 \% \mathrm{H}_{2} \mathrm{O} / 0.1 \%$ formic acid; buffer B: $100 \%$ acetonitrile $/ 0.1 \%$ formic acid). For each sample, cross-linking MS (XL-MS) data were acquired on a Thermo Orbitrap Elite using two similar data-dependent acquisition experiments ${ }^{50}$, where a single acquisition cycle consisted of: (1) one full $\mathrm{MS}^{1}$ (that is, parent/first) scan (at $350-1,500 \mathrm{~m} / z, 120,0000$ resolution, automatic gain control (AGC) target of $1 \times 10^{6}$ ); (2) followed by two data-dependent $\mathrm{MS}^{2}$ scans where the two most intense ions from the $\mathrm{MS}^{1}$ scan are isolated and fragmented (at 15,000 resolution, AGC target of $5 \times 10^{4}$, normalized collision energy $=22 \%$ ); and (3) followed by three (or four) data-dependent $\mathrm{MS}^{3}$ scans where the three-four most intense ions from the $\mathrm{MS}^{2}$ scan are isolated and fragmented (at ion count target $10^{4}$, normalized collision energy $=35 \%$ ). Precursor ions (charge state $\geq 4+$ ) were dynamically excluded for $20 \mathrm{~s}$ (tolerance of $10 \mathrm{ppm}$ ). Charge state and dynamic exclusion were applied to $\mathrm{MS}^{2}$ but turned off for $\mathrm{MS}^{3}$ acquisition.

Raw data were extracted to MGF format using MSConvert ${ }^{51}$, with MS $^{3}$ data used for protein and peptide searches. Searches were performed by the batch-tag feature of a locally installed version of Protein Prospector (v.5.19.1, University of California, San Francisco), with DSSO remnant mass modifications set as variable modifications (for example, alkene, sulfenic-acid and thiol) ${ }^{50}$. Peptide reports were generated using the Search Compare feature of Protein Prospector, and dead-end, intra-linked and inter-linked peptides were identified by in-house software program XL-Discoverer (part of the new XLTools suite) ${ }^{52}$. Summarization and confidence assignment of inter-linked peptides were performed by in-house scripts that reduce ambiguous assignments and distribute redundant counts. 
Integrative structure modeling of the Nef-CD4 $4_{\mathrm{CD}}-\mathrm{AP} 2^{\Delta \mu 2-C T D}$ complex We applied an integrative structural modeling approach ${ }^{34,53-55}$ to characterize the structure of the Nef-CD4 ${ }_{\mathrm{CD}}-\mathrm{AP} 2^{\triangle \mu 2-C T D}$ complex in solution, based on the crystal structure and the 90 DSSO cross-links. Integrative structure determination proceeded through the standard four stages ${ }^{53,54,56-59}$ : (1) gathering data; (2) representing subunits and translating data into spatial restraints; (3) configurational sampling to produce an ensemble of structures that satisfies the restraints; and (4) analyzing and validating the ensemble structures and data. The integrative structure modeling protocol (that is, stages 2, 3 and 4) was scripted using the Python Modeling Interface package, a library for modeling macromolecular complexes based on our open-source Integrative Modeling Platform package ${ }^{54}$, v.2.8 (https://integrativemodeling.org). Files containing the input data, scripts and output results are available at https://github.com/ integrativemodeling/Nef_CD4_AP2.

(1) Gathering data: Modeling was based on the crystal structure, a comparative model of the $\beta 2$ subunit $24-89$ region built based on the AP2 structure ${ }^{26}$ using MODELLER ${ }^{60,61}$ and the 90 DSSO cross-links.

(2) Representing subunits and translating data into spatial restraints: To maximize computational efficiency while avoiding using too coarse a representation, we represented the Nef-CD $4_{\mathrm{CD}}-\mathrm{AP} 2^{\triangle \mu 2-\mathrm{CTD}}$ complex using a coarse-grained one-residue-per-bead representation. The regions absent from the crystallographic structure and the comparative model were represented by a flexible string of beads corresponding to one residue each. To explore the positions and orientations of the Nef-CD4 $4_{\mathrm{CD}}-\mathrm{AP} 2^{\Delta \mu 2-\mathrm{CTD}}$ components, we defined the following rigid bodies: $\alpha-\sigma 2, \beta 2(89-591)-\mu 2$, Nef-CD $4_{C D}-\beta 2(15-23)$ and the four helices in the partially unfolded $\beta 2$ segment $(29-43,49-61$, 64-78 and 81-86). With this representation in hand, we next translated the input information into spatial restraints as follows.

First, the 90 DSSO cross-links were used to construct a Bayesian term that restrained the distances spanned by the cross-linked residues ${ }^{62,63}$. The cross-link restraints were applied to the one-residue-per-bead representation for the $\mathrm{X}$-ray structure, comparative models and flexible strings of beads. Second, to use the crystal structure of the hexamer as a template, we imposed 'structural equivalence' distance restraints between pairs of residues closer than $8.0 \AA$ across an interface between two rigid bodies, designed to restrain the model to resemble the template as much as possible. Third, excluded volume restraints were applied to all pairs of beads $s^{5,64}$. Fourth, we also applied the sequence connectivity restraint, using a harmonic upper bound on the distance between two consecutive beads in a subunit, with a threshold distance equal to four times the sum of the radii of the two connected beads ${ }^{56,64}$.

(3) Configurational sampling to produce an ensemble of structures that satisfies the restraints: The initial positions and orientations of rigid bodies and positions of the beads in the flexible strings of beads were randomized. The structural models were generated using replica exchange Gibbs sampling, based on the Metropolis Monte Carlo algorithm ${ }^{63,65}$. Each Monte Carlo step consisted of a series of random transformations (that is, rotations and translations) of the positions of the beads and rigid bodies. The sampling produced 4,000,000 models from 80 independent runs.

(4) Analyzing and validating the ensemble structures and data: Model validation follows four major steps ${ }^{34,66}$ :

i. Selection of the models for validation: The ensemble of models for further analysis was objectively defined as follows. For each trajectory, the Monte Carlo step at which all data likelihoods and priors are equilibrated (run equilibration step) was computed and all previous frames were discarded ${ }^{67}$. Sampling of the Nef-CD4 $4_{\mathrm{CD}}-\mathrm{AP} 2^{\triangle \mu 2-C T D}$ complex yielded $2,007,800$ representative structures that sufficiently satisfied the input restraints.

ii. Estimation of sampling precision: The precision at which sampling sampled the selected structures (sampling precision) was estimated ${ }^{66}$. The sampling precision must be comparable to or higher than the precision of the structure ensemble consistent with the input data (model precision). As a proxy for testing the thoroughness of sampling, we performed four sampling convergence tests as described ${ }^{66}$. We performed three sampling convergence tests: (1) We verified that the scores of refined structures did not continue to improve as more structures were computed. (2) We confirmed that the selected structures in independent sets of sampling runs (sample A and sample B) satisfied the data equally well. The non-parametric Kolmogorov-Smirnov two-sample test (two sided) indicated that the difference between the two score distributions was statistically insignificant $(P$ value $(0.08)>0.05)$. In addition, the magnitude of the difference $(D=0.02)$ was small, as demonstrated by the Kolmogorov-Smirnov two-sample test statistic. (3) We clustered the structural models and determined the sampling precision at which the structural features could be interpreted. Three criteria were used for determining the sampling precision, evaluated as a function of the r.m.s. deviation (r.m.s.d.) clustering threshold. First, the $P$ value was computed using the $\chi^{2}$-test (one-sided) for homogeneity of proportions. Second, an effect size for the $\chi^{2}$-test was quantified by the Cramer's $V$ value, a measure of association between two nominal variables. Third, the population of structures in sufficiently large clusters (containing at least ten structures from each sample). Clustering was done at the r.m.s.d. threshold at which three conditions were satisfied $\left(\chi^{2}\right.$-test $P(1.0)>0.05$, Cramer's $V$ $(0.0)<0.10$ and the population of clustered structures $(0.96)>0.80)$. The output of this protocol is a single distinct cluster containing the majority (96\%) of the individual models. The sampling precision is defined as the average bead r.m.s.d. between the models within the cluster and its corresponding centroid in the finest clustering, for which each sample contributes models proportionally to its size (considering both significance and magnitude of the difference) and for which a sufficient proportion of all models occur in sufficiently large clusters. The sampling precision for our integrative model is $13.5 \AA$.

iii.Estimation of model precision: The most explicit description of model uncertainty is provided by the set of all models that are sufficiently consistent with the input information (that is, the ensemble). For example, if the models in the ensemble are clustered into a single cluster as in the case of the Nef-CD4 $4_{\mathrm{CD}}-\mathrm{AP} 2^{\Delta \mu-\mathrm{CTD}}$ ensemble, the model precision is defined as the r.m.s.d. between models in the cluster. The precision for the Nef-CD $4_{\mathrm{CD}^{-}}$ $\mathrm{AP} 2^{\triangle \mathrm{M2}-\mathrm{CTD}}$ model is $8.3 \AA$.

iv. Quantification of the degree to which a model satisfies the information used to compute it: An accurate structure needs to satisfy the input information used to compute it. A DSSO cross-link restraint is satisfied by a cluster of models if the corresponding $\mathrm{C} \alpha-\mathrm{C} \alpha$ distance in any of the models is less than $30 \AA$ (ref. $\left.{ }^{68}\right)$. The ensemble satisfies $89 \%$ of the cross-links used to compute it, including all of the Nef cross-links (Extended Data Fig. 3). The unsatisfied cross-links mostly span residues between $\mathrm{CD} 4_{\mathrm{CD}}$ and the NTDs of the $\alpha$ and $\beta 2$ subunits (Supplementary Table 1). These violations can be rationalized as nonspecific interactions between $\mathrm{CD}$ $4_{\mathrm{CD}}$ and the AP2 complex when $\mathrm{CD} 4_{\mathrm{CD}}$ is not bound to Nef in solution, false-positive cross-links, sample heterogeneity, insufficient conformational sampling and coarse-grained representation of the modeled components. The remaining restraints, including structural equivalence, excluded volume and sequence connectivity restraints, are also satisfied within their uncertainties.

To compare the X-ray structure with the ensemble obtained using integrative modeling, we computed the distribution of the $\mathrm{C} \alpha$ r.m.s.d. between the X-ray structure and each of the models in the ensemble. The mean C $\alpha$ r.m.s.d. is $7.1 \AA$ (95\% confidence interval, $4.3-12.2$ ).

To indicate the most flexible parts of the structure, we assessed the uncertainty of the position and orientation of each rigid body representing the hexamer in the model ensemble. To this end, all models were superimposed on each rigid body in turn, followed by computing the average r.m.s.d. for each of the other rigid bodies (Extended Data Fig. 3e). The model ensemble indicates large variability in the positions and orientations of the four helices in the partially unfolded $\beta 2$ segment (Extended Data Fig. 3e,g), consistent with the structural heterogeneity of this region indicated by the relative lack of electron density from crystallography.

Reporting Summary. Further information on research design is available in the Nature Research Reporting Summary linked to this article.

\section{Data availability}

The coordinates and structure factors for the crystal structure have been deposited at the Protein Data Bank (PDB) with the accession code 6URI. The proteomics XL-MS data have been deposited at the ProteomeXchange database ${ }^{69}$ with the accession code PXD019338. The integrative structural model has been deposited at PDB-Dev with the accession code PDBDEV_00000050. Source data are provided with this paper.

\section{Code availability}

Files containing the input data, scripts and output results for the integrative structure modeling of the Nef-CD4 $4_{\mathrm{CD}}-\mathrm{AP} 2^{\Delta \mu 2-\mathrm{CTD}}$ complex are available at https:// github.com/integrativemodeling/Nef_CD4_AP2.

\section{References}

43. Xue, X. Y. et al. Production of authentic SARS-CoV M-pro with enhanced activity: application as a novel tag-cleavage endopeptidase for protein overproduction. J. Mol. Biol. 366, 965-975 (2007).

44. Xue, X. Y. et al. Structures of two coronavirus main proteases: implications for substrate binding and antiviral drug design. J. Virol. 82, 2515-2527 (2008).

45. Otwinowski, Z. \& Minor, W. Processing of X-ray diffraction data collected in oscillation mode. Methods Enzymol. 276, 307-326 (1997).

46. McCoy, A. J. et al. Phaser crystallographic software. J. Appl. Crystallogr. 40, 658-674 (2007).

47. Adams, P. D. et al. PHENIX: a comprehensive Python-based system for macromolecular structure solution. Acta Crystallogr. D Biol. Crystallogr. 66 , 213-221 (2010). 
48. Emsley, P. \& Cowtan, K. Coot: model-building tools for molecular graphics. Acta Crystallogr. D Biol. Crystallogr. 60, 2126-2132 (2004).

49. Afonine, P. V. et al. Towards automated crystallographic structure refinement with phenix.refine. Acta Crystallogr. D Biol. Crystallogr. 68, 352-367 (2012).

50. Kaake, R. M. et al. A new in vivo cross-linking mass spectrometry platform to define protein-protein interactions in living cells. Mol. Cell. Proteom. 13, 3533-3543 (2014).

51. Kessner, D., Chambers, M., Burke, R., Agus, D. \& Mallick, P. ProteoWizard: open source software for rapid proteomics tools development. Bioinformatics 24, 2534-2536 (2008).

52. Gutierrez, C. B. et al. Developing an acidic residue reactive and sulfoxide-containing MS-cleavable homobifunctional cross-linker for probing protein-protein interactions. Anal. Chem. 88, 8315-8322 (2016).

53. Kim, S. J. et al. Integrative structure and functional anatomy of a nuclear pore complex. Nature 555, 475-482 (2018).

54. Russel, D. et al. Putting the pieces together: integrative modeling platform software for structure determination of macromolecular assemblies. PLoS Biol. 10, e1001244 (2012).

55. Ward, A. B., Sali, A. \& Wilson, I. A. Integrative structural biology. Science 339, 913-915 (2013).

56. Alber, F. et al. Determining the architectures of macromolecular assemblies. Nature 450, 683-694 (2007).

57. Lasker, K. et al. Molecular architecture of the $26 \mathrm{~S}$ proteasome holocomplex determined by an integrative approach. Proc. Natl Acad. Sci. USA 109, 1380-1387 (2012)

58. Sali, A. et al. Outcome of the first wwPDB Hybrid/Integrative Methods Task Force Workshop. Structure 23, 1156-1167 (2015).

59. Schneidman-Duhovny, D., Pellarin, R. \& Sali, A. Uncertainty in integrative structural modeling. Curr. Opin. Struct. Biol. 28, 96-104 (2014).

60. Sali, A. \& Blundell, T. L. Comparative protein modelling by satisfaction of spatial restraints. J. Mol. Biol. 234, 779-815 (1993).

61. Webb, B. \& Sali, A. Comparative protein structure modeling using MODELLER. Curr. Protoc. Protein Sci. 54, 5.6.1-5.6.37 (2016).

62. Erzberger, J. P. et al. Molecular architecture of the 40S-eIF1.eIF3 translation initiation complex. Cell 159, 1227-1228 (2014).

63. Shi, Y. et al. Structural characterization by cross-linking reveals the detailed architecture of a coatomer-related heptameric module from the nuclear pore complex. Mol. Cell. Proteom. 13, 2927-2943 (2014).

64. Shen, M. Y. \& Sali, A. Statistical potential for assessment and prediction of protein structures. Protein Sci. 15, 2507-2524 (2006).

65. Swendsen, R. H. \& Wang, J. S. Replica Monte Carlo simulation of spin glasses. Phys. Rev. Lett. 57, 2607-2609 (1986)

66. Viswanath, S., Chemmama, I. E., Cimermancic, P. \& Sali, A. Assessing exhaustiveness of stochastic sampling for integrative modeling of macromolecular structures. Biophys. J. 113, 2344-2353 (2017).
67. Chodera, J. D. A simple method for automated equilibration detection in molecular simulations. J. Chem. Theory Comput. 12, 1799-1805 (2016).

68. Merkley, E. D. et al. Distance restraints from crosslinking mass spectrometry: mining a molecular dynamics simulation database to evaluate lysine-lysine distances. Protein Sci. 23, 747-759 (2014).

69. Perez-Riverol, Y, et al. The PRIDE database and related tools and resources in 2019: improving support for quantification data. Nucleic Acids Res. 47, D442-D450 (2019).

\section{Acknowledgements}

We thank Y. Xiong (Yale University) for helpful discussions and valuable input. We thank the beamline staff at the Advanced Photon Source beamline 24-ID and the National Synchrotron Light Source beamline 17-ID. We thank J. Bonifacino (National Institutes of Health (NIH)) for providing the gene of rat $\alpha$ adaptin. This work was supported by the University of Massachusetts Dartmouth startup fund (X.J.) and US NIH grants no. AI102778 and no. AI129706 (J.G.). R.M.K., I.E., A.S. and N.K. were supported by NIH grant no. P50AI150476. R.M.K. was also supported by NIH fellowship grant no. F32AI127291. A.S. was also supported by NIH grants no. U19AI135990, no. R01GM083960, no. P41GM109824 and no. S10OD021596. N.K. was also supported by NIH grants no. P50GM082250 and no. U19AI135990.

\section{Author contributions}

Y.K. performed protein expression, purification, binding assays and crystallization. Y.K. and X.J. performed data collection, structure determination, model building and refinement. M.S., C.S. and P.W.R. performed CD4 and MHC-I downregulation assays and mutagenesis. R.M.K. performed XL-MS. I.E. performed integrative modeling. Y.K and M.K.S. performed in vitro mutagenesis and fluorescence polarization assays. J.K. and R.S. contributed to protein expression and purification. Y.K., R.M.K., I.E., A.S., N.K., J.G. and X.J. designed the experiments. All contributed to data analysis. J.G. and X.J. supervised the project. Y.K., J.G. and X.J. wrote the manuscript.

\section{Competing interests}

The authors declare no competing interests.

\section{Additional information}

Extended data is available for this paper at https://doi.org/10.1038/s41594-020-0463-z. Supplementary information is available for this paper at https://doi.org/10.1038/ s41594-020-0463-Z.

Correspondence and requests for materials should be addressed to X.J.

Peer review information Peer reviewer reports are available. Inês Chen was the primary editor on this article and managed its editorial process and peer review in collaboration with the rest of the editorial team.

Reprints and permissions information is available at www.nature.com/reprints. 


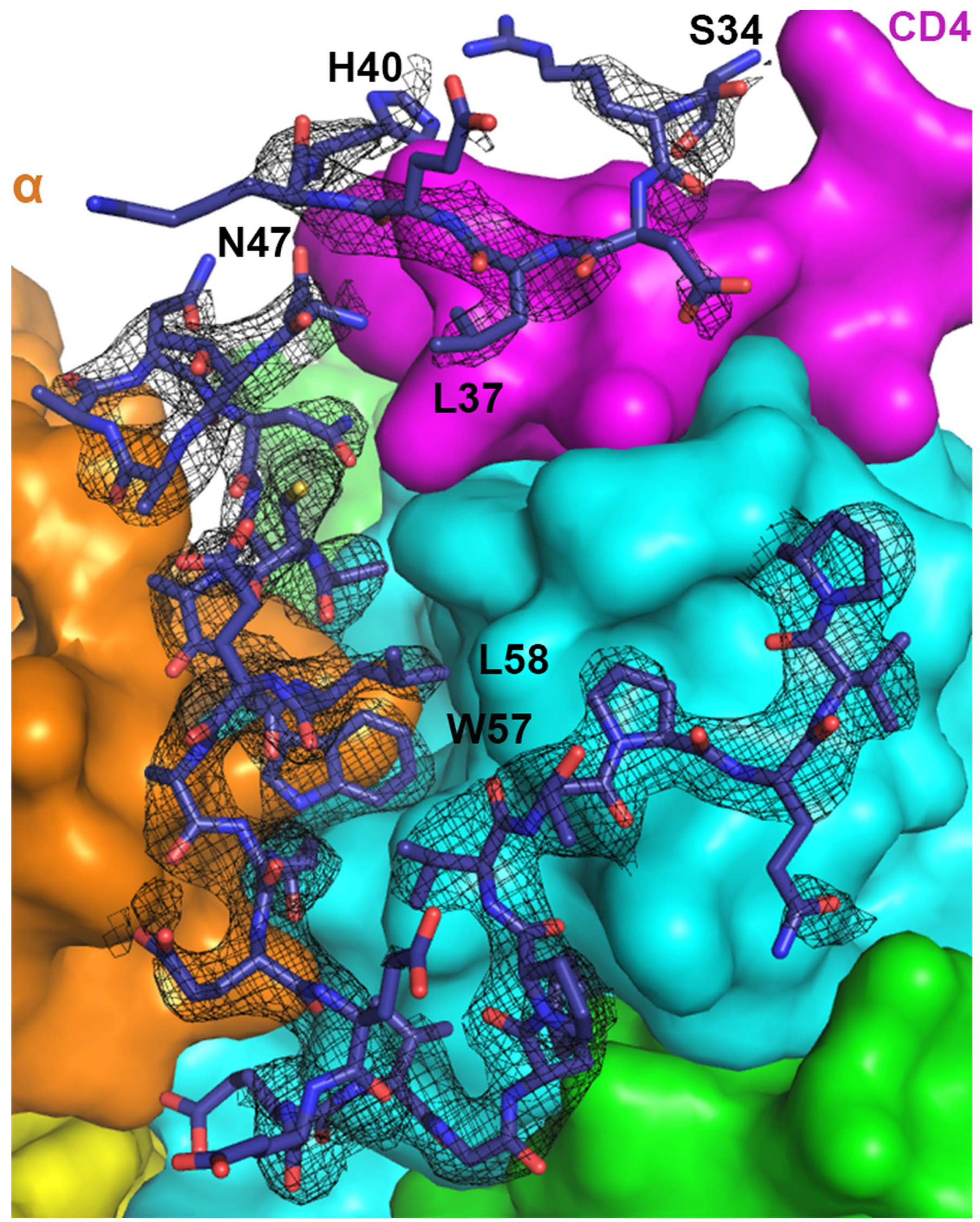

Extended Data Fig. 1 | Electron density map for the N-terminal loop of Nef. 2Fo-Fc map (1 $\sigma$ level with B factor sharpened by $-50 \AA^{2}$ ) for Nef residues 34-40 and 47-75 is shown as black mesh. Nef residues 41-46 could not be built due to the lack of density. Density for Nef 34-40 is less defined, although sidechain density for Leu37 is clear. 


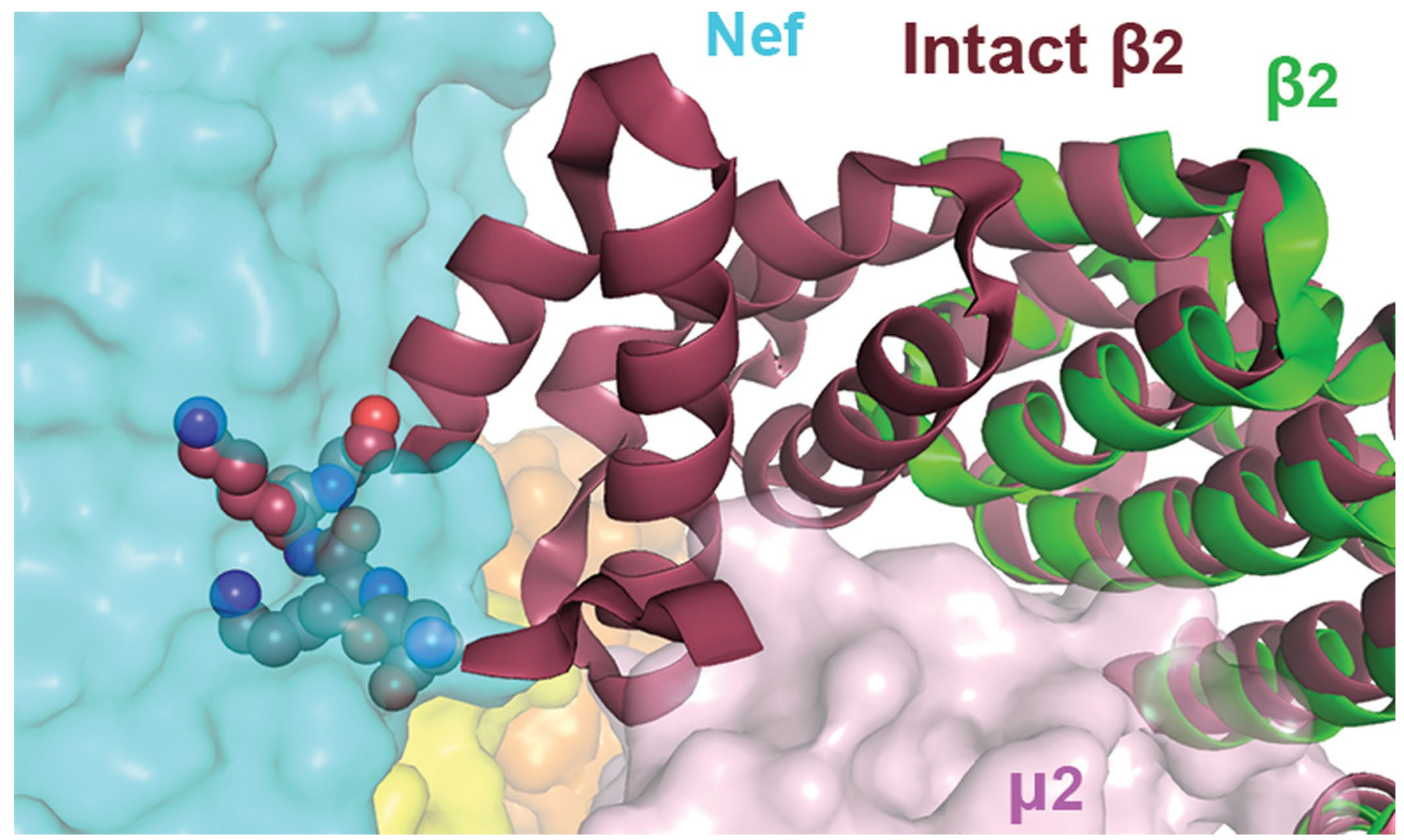

Extended Data Fig. 2 | $\beta 2$ subunit, if intact, would clash with the bound Nef. Overlay of the intact $\beta 2$ subunit (dark red, PDB 2XA7) with $\beta 2$ in the current structure (green) indicates that clashing would take place between Nef and N-terminus of the intact $\beta 2$, specifically residues Asn10, Lys11, Lys12, and Gly13 (spheres). 
a

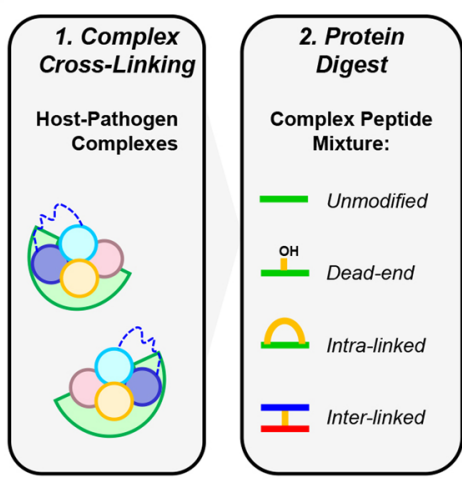

b

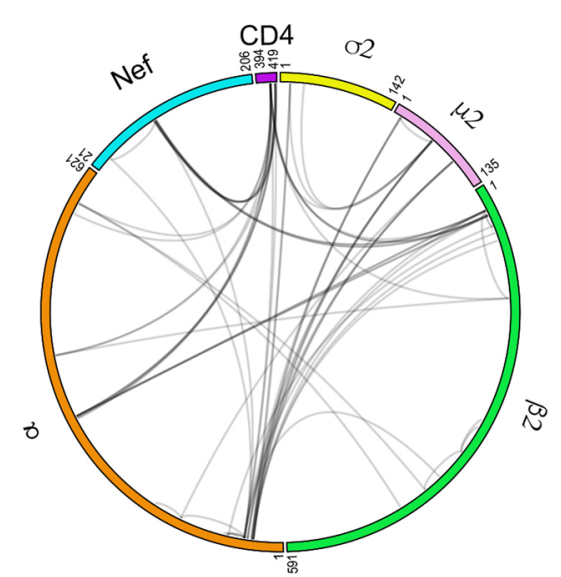

e

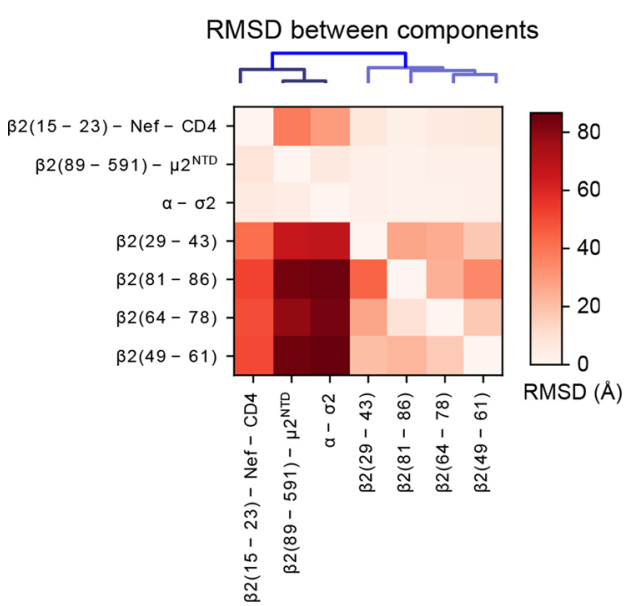

C
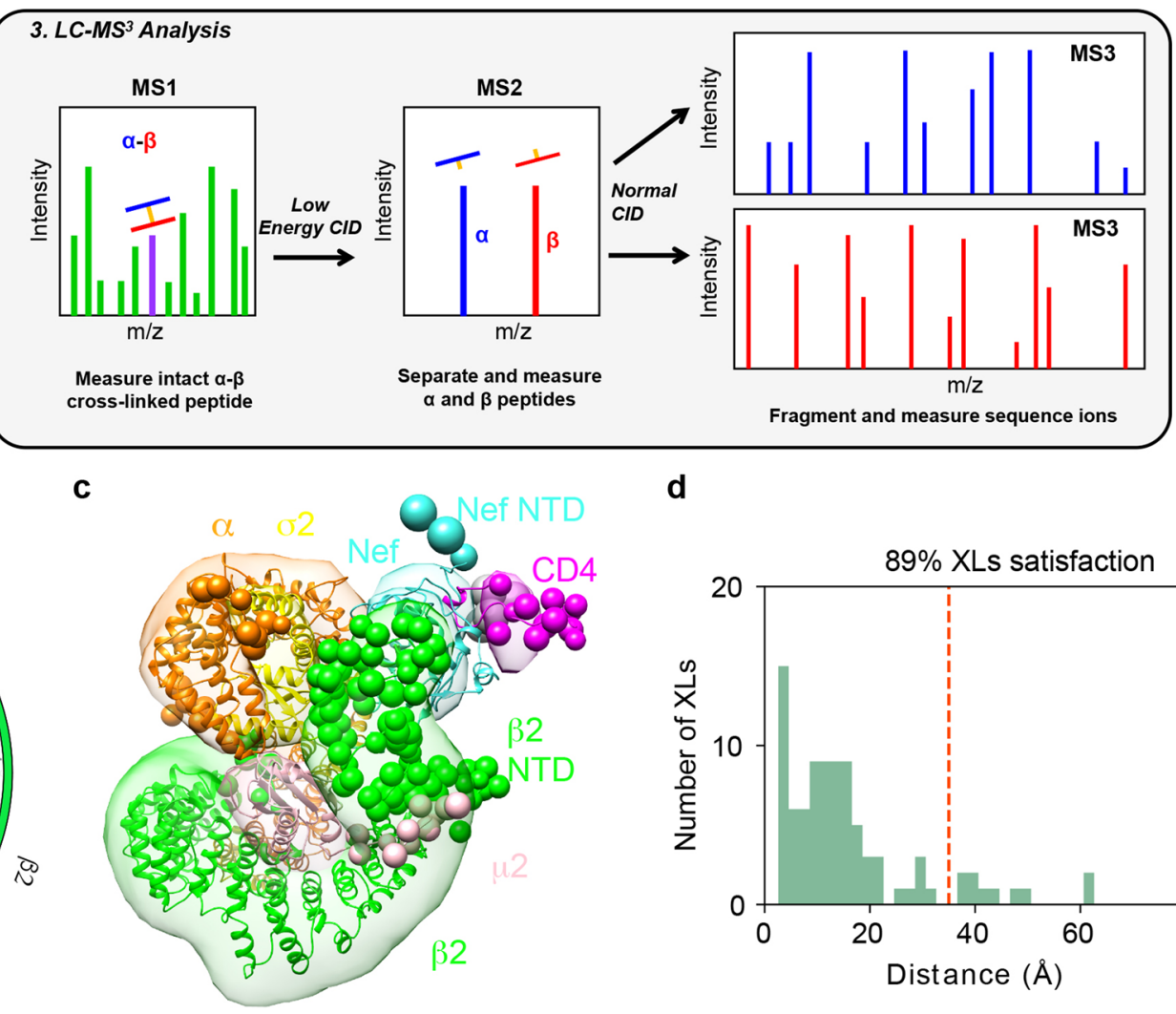

d

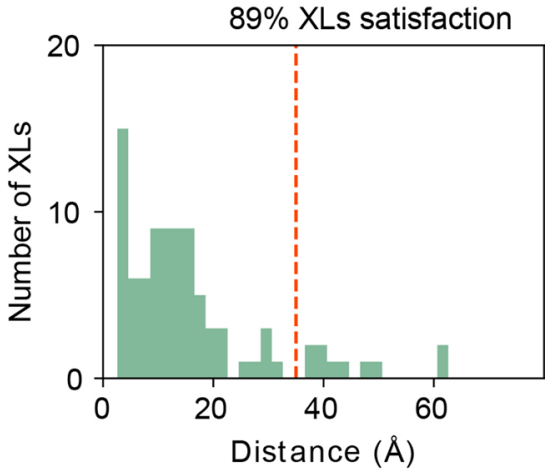

g

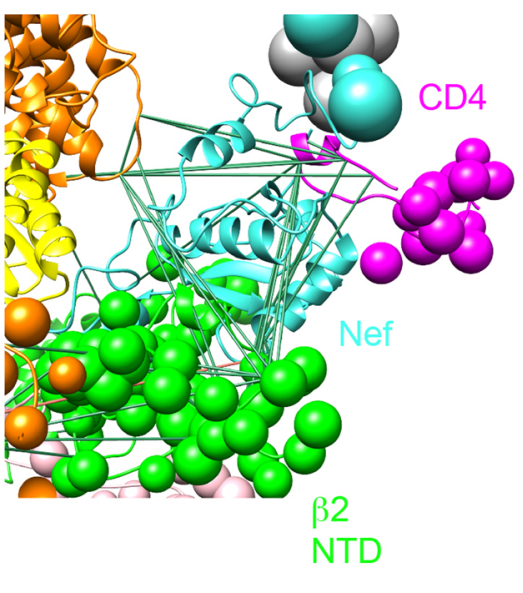

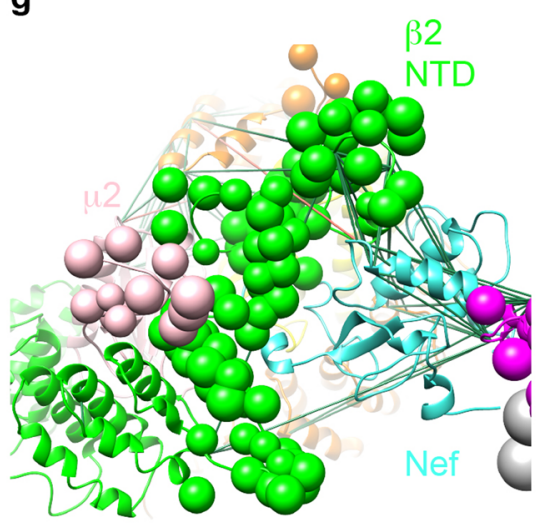

g

Extended Data Fig. 3 | Crosslinking mass spectrometry and integrative structure modeling of the Nef-CD4 ${ }_{C D}-A P 2^{\Delta \mu 2-C T D}$ complex. a, Overview of the DSSO XL-MS ${ }^{3}$ analysis method. b, CX-Circos linkage map of all Nef-CD4 $4_{C D}-A P 2^{\triangle \mu 2-C T D}$ interlinks. c, Integrative structure of the Nef-CD4 ${ }_{C D}-A P 2^{\triangle \mu 2-C T D}$ complex. The localization probability density of the ensemble of structures is shown with representative (centroid) structure from the ensemble embedded within it. Regions present in the crystal structure are shown as ribbons and segments not present in the crystal structure are shown as beads. d, Histogram showing the distribution of the cross-linked $\mathrm{C} \alpha-\mathrm{C} \alpha$ distances in the integrative structure. The structural ensemble satisfies $89 \%$ of the XLs used to compute it. e, RMSD between rigid-bodies in the model ensemble. The vertical axis corresponds to the rigid body used as reference for superimposition and the horizontal axis are the rigid bodies for which the average RMSD was computed. f, Detail of crosslinks mapped to Nef. Satisfied and violated crosslinks shown in green and pink, respectively. $\mathbf{g}$, Positioning of the unfolded $\beta 2$ segment. 


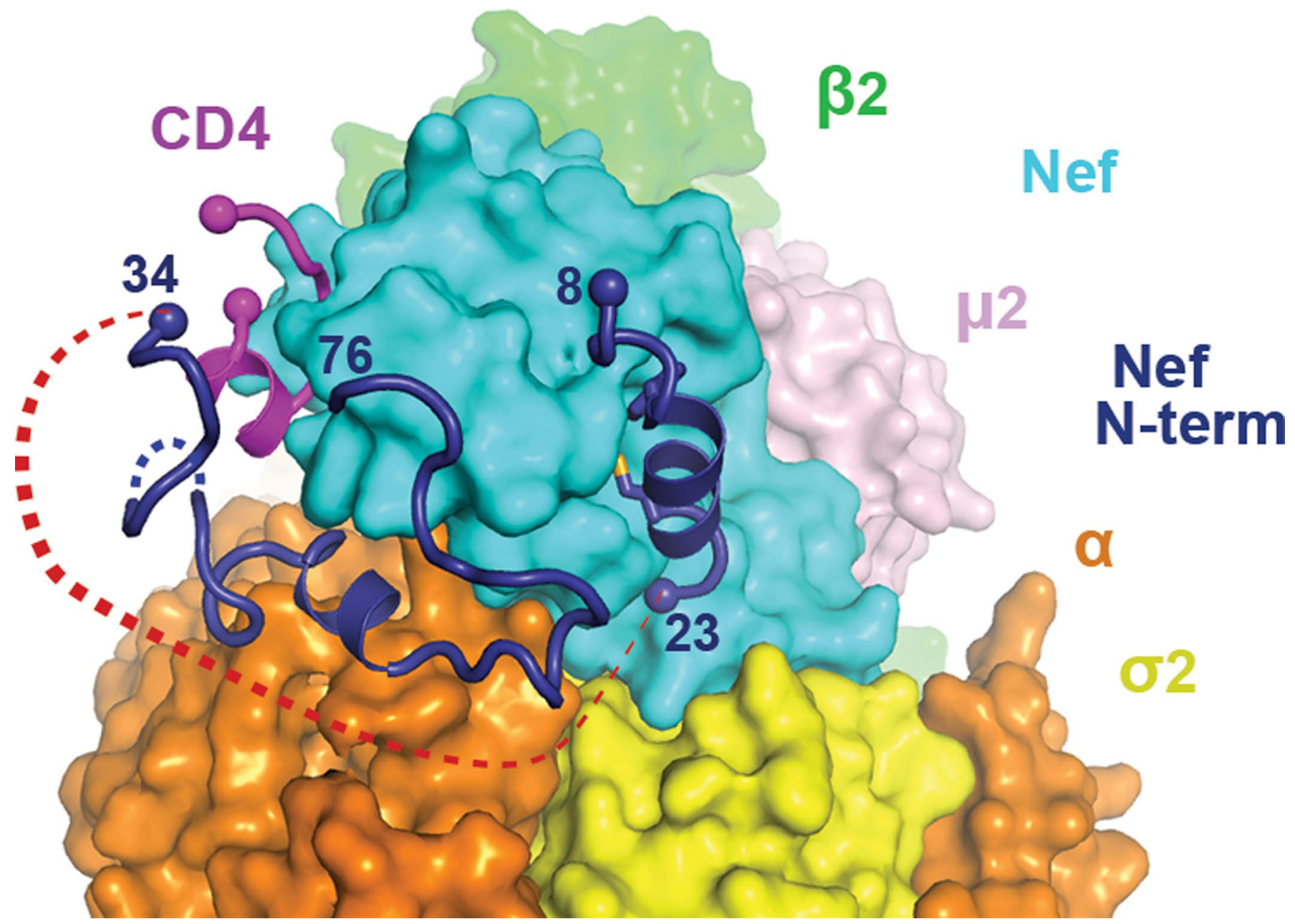

Extended Data Fig. 4 | Binding of Nef N-terminal helix to the Nef core is incompatible with CD4 downregulation. N-terminal helix of Nef (8-23) is modeled into the current structure. Red dotted line represents the would-be distance between residues 23 and 34 , which cannot be covered by ten residues (Nef 24-33). 


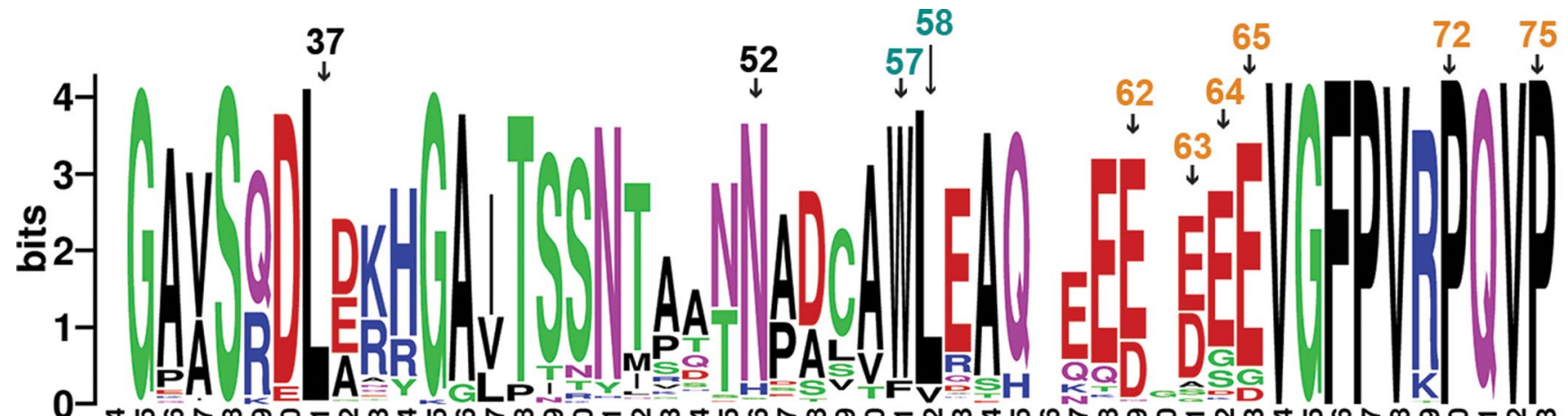

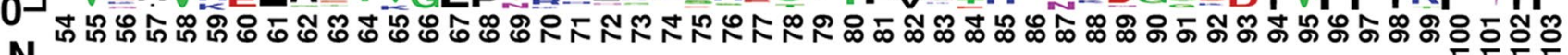

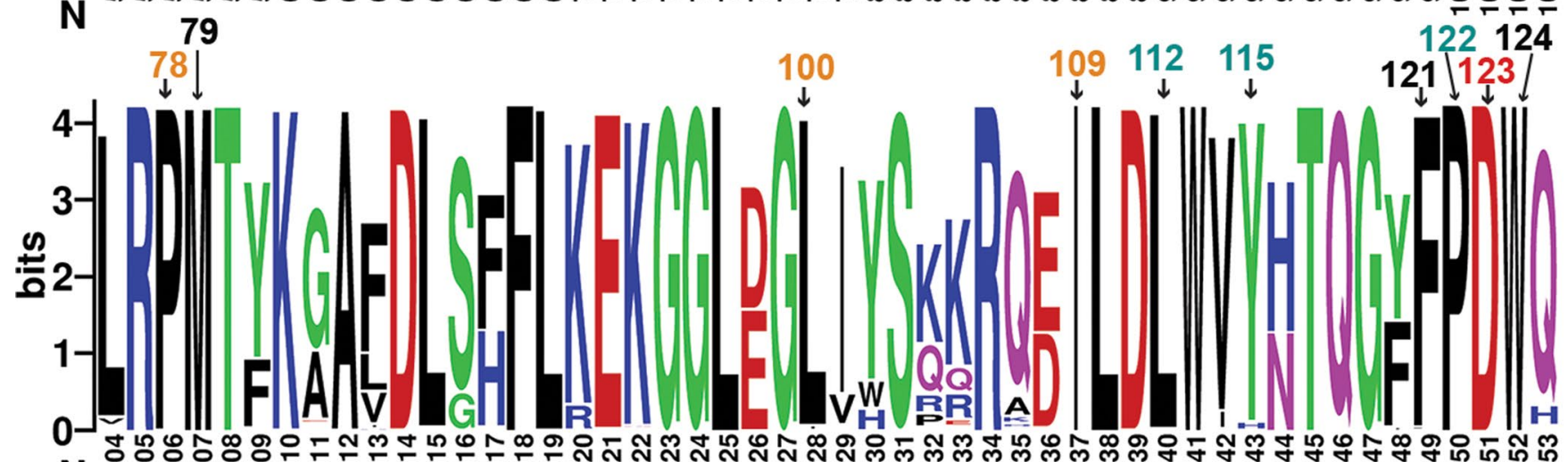

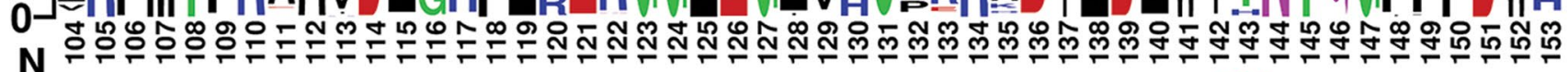

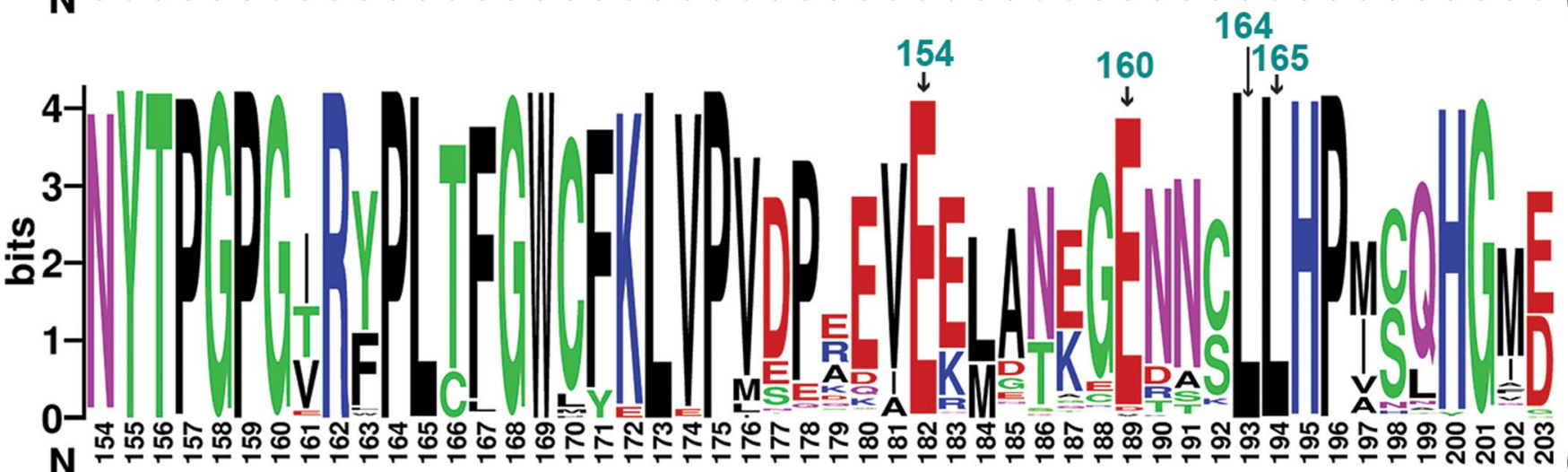

weblogo.berkeley.edu

Extended Data Fig. 5 | Nef residues at the CD4 -binding pocket are highly conserved. Nef sequences from HIV sequence compendium 2017 were analyzed though multiple sequence alignment (HIV sequence database, www.hiv.lanl.gov). Alignment was done in HXB2 convention (bottom) and important residues are additionally labeled using the NL4.3 convention on top. D123, shown in red text, is important for both CD4 and MHC-I downregulation. Other residues important for CD4 downregulation are in cyan and black texts. Black texts refer to residues, in addition to D123, that surround CD4. Other residues important for MHC-I downregulation are in orange. The logo representation, with the height of each letter proportional to the observed frequency of the corresponding amino acid residue, was generated by WebLogo ${ }^{70}$. 

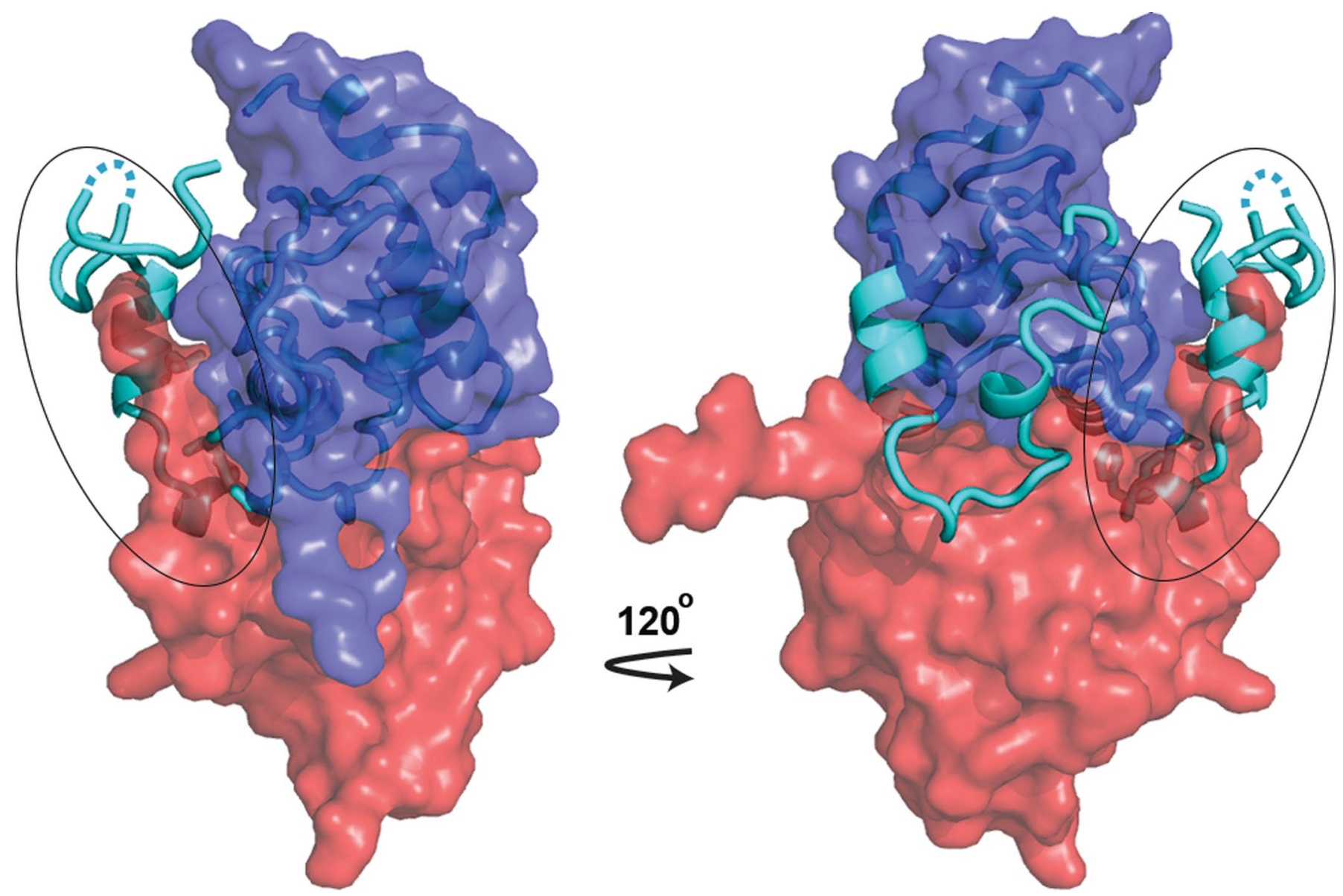

Extended Data Fig. 6 | The unique conformation of Nef N-terminal loop observed in the current structure is incompatible with Nef dimerization. Nef in current conformation (cyan, cartoon) is overlaid with the $\mathrm{SH} 2-\mathrm{SH} 3$-dependent Nef dimer ${ }^{39}$ (dark blue and red envelopes, PDB 4U5W). While majority of Nef in the current structure overlays well with the Nef protomer shown as the dark blue envelope, the N-terminal region of Nef (circled) intrudes severely into the volume of the other Nef protomer (red envelope). 


\section{Reporting Summary}

Nature Research wishes to improve the reproducibility of the work that we publish. This form provides structure for consistency and transparency in reporting. For further information on Nature Research policies, see Authors \& Referees and the Editorial Policy Checklist.

\section{Statistics}

For all statistical analyses, confirm that the following items are present in the figure legend, table legend, main text, or Methods section. $\mathrm{n} / \mathrm{a}$ Confirmed

\ The exact sample size $(n)$ for each experimental group/condition, given as a discrete number and unit of measurement

\ A statement on whether measurements were taken from distinct samples or whether the same sample was measured repeatedly

$\triangle$ The statistical test(s) used AND whether they are one- or two-sided

Only common tests should be described solely by name; describe more complex techniques in the Methods section.

Х $\square$ A description of all covariates tested

Х $\square$ A description of any assumptions or corrections, such as tests of normality and adjustment for multiple comparisons A full description of the statistical parameters including central tendency (e.g. means) or other basic estimates (e.g. regression coefficient) AND variation (e.g. standard deviation) or associated estimates of uncertainty (e.g. confidence intervals) For null hypothesis testing, the test statistic (e.g. $F, t, r)$ with confidence intervals, effect sizes, degrees of freedom and $P$ value noted Give $P$ values as exact values whenever suitable.

Х $\square$ For Bayesian analysis, information on the choice of priors and Markov chain Monte Carlo settings

$\square$ For hierarchical and complex designs, identification of the appropriate level for tests and full reporting of outcomes

$\square \bigotimes$ Estimates of effect sizes (e.g. Cohen's $d$, Pearson's $r$ ), indicating how they were calculated

\section{Our web collection on statistics for biologists contains articles on many of the points above.}

\section{Software and code}

Policy information about availability of computer code

Data collection

Provide a description of all commercial, open source and custom code used to collect the data in this study, specifying the version used OR state that no software was used.

Data analysis

Provide a description of all commercial, open source and custom code used to analyse the data in this study, specifying the version used OR state that no software was used.

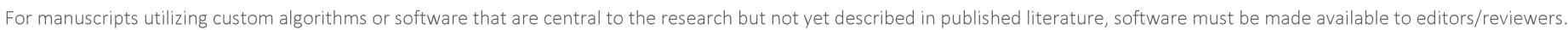
We strongly encourage code deposition in a community repository (e.g. GitHub). See the Nature Research guidelines for submitting code \& software for further information.

\section{Data}

Policy information about availability of data

All manuscripts must include a data availability statement. This statement should provide the following information, where applicable:

- Accession codes, unique identifiers, or web links for publicly available datasets

- A list of figures that have associated raw data

- A description of any restrictions on data availability

The coordinates and structural factors for the crystal structure have been deposited at the Protein Data Bank (PDB) with the accession code 6URI.

\section{Field-specific reporting}

Please select the one below that is the best fit for your research. If you are not sure, read the appropriate sections before making your selection. 


\section{Life sciences study design}

All studies must disclose on these points even when the disclosure is negative.

Sample size n/a
Data exclusions n/a
Replication $\mathrm{n} / \mathrm{a}$
Randomization
Blinding

\section{Reporting for specific materials, systems and methods}

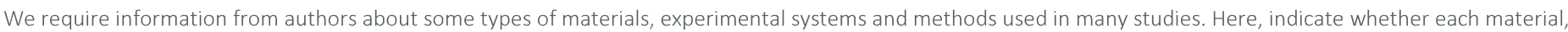

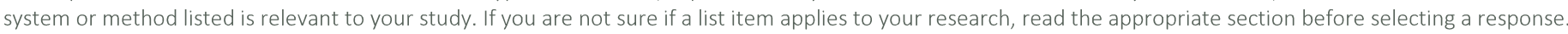

\begin{tabular}{|c|c|c|c|}
\hline \multicolumn{2}{|c|}{ Materials \& experimental systems } & \multicolumn{2}{|c|}{ Methods } \\
\hline $\mathrm{n} / \mathrm{a}$ & Involved in the study & $\mathrm{n} / \mathrm{a}$ & Involved in the study \\
\hline & \ Antibodies & Х & $\square$ ChIP-seq \\
\hline & 区ukaryotic cell lines & & \ Flow cytometry \\
\hline Х & Palaeontology & Х & MRI-based neuroimaging \\
\hline Х & Animals and other organisms & & \\
\hline X & Human research participants & & \\
\hline X & Clinical data & & \\
\hline
\end{tabular}

\section{Antibodies}

Antibodies used

Validation anti-human CD4 (Biolegend, San Diego CA, USA).; anti-HLA-A2 (Biolegend, San Diego, CA, USA).

per manufacturer

\section{Eukaryotic cell lines}

\section{Policy information about cell lines}

Cell line source(s)

HeLa-TZM-bl (Dr. John Kappes via the NIH AIDS Reagent Program) and HEK293 (Dr. Celsa Spina UCSD originally from Dr. Joseph Nevins, Duke University).

\section{Authentication \\ Mycoplasma contamination \\ Commonly misidentified lines (See $\underline{I C L A C}$ register)}

not authenticated

excluded by PCR assay

Name any commonly misidentified cell lines used in the study and provide a rationale for their use.

\section{Flow Cytometry}

Plots

Confirm that:

$\square$ The axis labels state the marker and fluorochrome used (e.g. CD4-FITC).

\The axis scales are clearly visible. Include numbers along axes only for bottom left plot of group (a 'group' is an analysis of identical markers).

$\square$ All plots are contour plots with outliers or pseudocolor plots.

$\bigotimes$ A numerical value for number of cells or percentage (with statistics) is provided. 
Cells (HEK293 or HeLa-TZM-bl) were removed from the plates and incubated with APC-conjugated antibody to HAL-A2 (HEK239) or APC-conjugated antibody to CD4 (HeLa-TZM-bl) or APC-conjugated antibody isotype controls. After washing to remove unbound antibody, the cells were fixed with $1 \%$ formaldehyde before analysis.

Instrument

Accuri A6 benchtop cytometer.

\section{Software}

Cell population abundance

CFlow Plus.

The "live cell gate" was determined empirically based on forward scatter and side-scatter characteristics using cells that were not transfected but were fixed with $1 \%$ formaldehyde. 10,000 events (cells) in the live cell gate were used to generate the two-color data presented (CD4-APC or HLA-A2-APC vs. GFP).

Gating strategy

The live cell gates were determined using non-transfected but formaldehyde-fixed fixed cells and were applied to all the transfected cell populations. The boundaries for GFP-positive cells were set using cells not transfected to express GFP. The boundaries for CD4-postive or HLA-A2-positive cells were set using APC-conjugated antibody isotype controls.

Tick this box to confirm that a figure exemplifying the gating strategy is provided in the Supplementary Information. 